Article

\title{
Estimating the Lower Limit of the Impact of Amines on Nucleation in the Earth's Atmosphere
}

\author{
Alexey B. Nadykto ${ }^{1,2,3, *}$, Jason Herb ${ }^{2}$, Fangqun $\mathrm{Yu}^{2}$, Yisheng $\mathrm{Xu}{ }^{1,2, *}$ and \\ Ekaterina S. Nazarenko ${ }^{3}$
}

1. State Key Laboratory of Environmental Criteria and Risk Assessment, Chinese Research Academy of Environmental Sciences, Beijing 100012, China

2. Atmospheric Science Research Center, State University of New York at Albany, 251 Fuller Road, Albany, NY 12203, USA; E-Mails: jherb@albany.edu (J.H.); fyu@albany.edu (F.Y.)

3. Department of Applied Mathematics, Moscow State University of Technology "Stankin", Vadkovsky per. 1, Moscow 127055, Russia; E-Mail: katarina.86@bk.ru

* Author to whom correspondence should be addressed: E-Mails: anadykto@albany.edu (A.B.N); xuys@craes.org.cn (Y.X.); Tel.: +1-518-437-8732 (A.B.N); +86-10-84915249 (Y.X.); Fax: +1-518-437-8756 (A.B.N); +86-10-84915248 (Y.X.).

Academic Editor: Kevin H. Knuth

Received: 27 January 2015 / Accepted: 20 April 2015 / Published: 30 April 2015

\begin{abstract}
Amines, organic derivatives of $\mathrm{NH}_{3}$, are important common trace atmospheric species that can enhance new particle formation in the Earth's atmosphere under favorable conditions. While methylamine (MA), dimethylamine (DMA) and trimethylamine (TMA) all efficiently enhance binary nucleation, MA may represent the lower limit of the enhancing effect of amines on atmospheric nucleation. In the present paper, we report new thermochemical data concerning MA-enhanced nucleation, which were obtained using the DFT PW91PW91/6-311++G (3df, 3pd) method, and investigate the enhancement in production of stable pre-nucleation clusters due to the MA. We found that the MA ternary nucleation begins to dominate over ternary nucleation of sulfuric acid, water and ammonia at $[\mathrm{MA}] /\left[\mathrm{NH}_{3}\right]>\sim 10^{-3}$. This means that under real atmospheric conditions ([MA] $\sim 1 \mathrm{ppt}$, $\left[\mathrm{NH}_{3}\right] \sim 1 \mathrm{ppb}$ ) the lower limit of the enhancement due to methylamines is either close to or higher than the typical effect of $\mathrm{NH}_{3}$. A very strong impact of the MA is observed at low $\mathrm{RH}$; however it decreases quickly as the RH grows. Low RH and low ambient temperatures were found to be particularly favorable for the enhancement in production of stable sulfuric acid-water clusters due to the MA.
\end{abstract}


Keywords: new particle formation; amines; ammonia; nucleation; DFT; thermochemistry

\section{Introduction}

Nucleation is a critically important process that significantly increases concentrations of cloud condensation nuclei $(\mathrm{CCN})$ and impacts the Earth's climate due to scattering and absorbtion of solar radiation [1-4]. Formation of new nano- and ultrafine particles in polluted areas of the planetary boundary layer (PBL) causes adverse public health impacts, including epidemics of respiratory and cardiovascular diseases, lung cancer and increased overall mortality due to the presence of toxic species in inhaled particles [5,6]. Although the origin and chemical composition of new atmospheric nanoparticles have been studied for decades, there still exist large uncertainties concerning the impacts of biogenic and anthropogenic emissions on the Earth's climate related to new particle formation. Atmospheric nucleation rates correlate with $\left[\mathrm{H}_{2} \mathrm{SO}_{4}\right]$ and are observed only if $\left[\mathrm{H}_{2} \mathrm{SO}_{4}\right]>10^{5} \mathrm{~cm}^{-3}$ [7]. However, binary $\mathrm{H}_{2} \mathrm{SO}_{4}-\mathrm{H}_{2} \mathrm{O}(\mathrm{BHN})[8,9]$ nucleation cannot fully explain nucleation events observed in the polluted PBL $[1-4,7,10]$. While key atmospheric nucleation mechanisms are still a subject of ongoing debates [1-5,7,11-14], it is commonly accepted that trace atmospheric species other than $\mathrm{H}_{2} \mathrm{SO}_{4}$ and $\mathrm{H}_{2} \mathrm{O}$ are involved in new particle formation in the Earth's atmosphere and that neutral $\mathrm{H}_{2} \mathrm{SO}_{4}-\mathrm{H}_{2} \mathrm{O}$ clusters must be stabilized with ions [12,13], ammonia [14-19], amines [20-23] or organic acids [24-32] in order to nucleate. Theoretical formalism of nucleation of $\mathrm{H}_{2} \mathrm{SO}_{4}, \mathrm{H}_{2} \mathrm{O}$ and $\mathrm{NH}_{3}$ is commonly known as the Ternary Homogeneous Nucleation (THN) theory [11,14-19]. The theory has been developed into a global concept explaining nucleation in the PBL back in the early 2000 s. However, the THN prediction [11,15] of an enhancement of up to $\sim 10^{30}$ atmospheric nucleation rates due to a few ppt of $\mathrm{NH}_{3}$ later failed due to mistakes in nucleation thermodynamics and kinetics [16,17] and disagreement with laboratory studies showing much smaller $\mathrm{NH}_{3}$ effects of up to a few orders of magnitude at $\left[\mathrm{NH}_{3}\right]>1 \mathrm{ppb}[14,18,19]$.

Amines, organic derivatives of ammonia, are likely to be the strongest bases that are present in the Earth's atmosphere in considerable concentrations. They are present in form of the gas phase molecules, particulate matter and cloud and fog droplets [33,34]. Common atmospheric sources of ammonia and amines include vegetation, biomass burning, animal farms, cooking, vehicular emissions, and various industrial processes [33,35]. Amines can cause adverse public health impacts $[36,37]$ due to the rapid oxidation of amines to carcinogens such as nitrosamines and isocyanic acid [38,39]. Out of a number of amines detected in the Earth's atmosphere [33,36,37], aliphatic amines-methylamine MA, dimethylamine DMA and trimethylamine TMA - are the most well-studied ones. The background concentrations of aliphatic amines and ammonia are typically of the order of ppt to tens of ppt [34,40-42] and sub-ppb to tens of ppb [43-45], respectively. It is usually assumed that typical atmospheric concentrations of aliphatic amines are ranked in the order $[\mathrm{MA}]<[\mathrm{DMA}]<[\mathrm{TMA}]$. However, the actual atmospheric concentrations of MA, DMA and TMA may vary depending on the location and season. For example, in the most recent study of atmospheric concentrations of MA, DMA, and TMA You et al. [33] reported [MA] 1-4 pptv and <0.1 pptv and [TMA] 5-10 pptv and 1-10 pptv at Kent and an Alabama forest, respectively, while [DMA] at both sites was found to be below the detection 
limit of $0.5 \mathrm{pptv}$. Interested readers can learn more about amines from a recent review on the multiphase chemistry of atmospheric amines, which contains comprehensive discussions on the role of amines in aerosol nucleation and growth [51].

Laboratory nucleation studies show that amines are more than likely to enhance binary nucleation and that the enhancement due to amines depends on amine basicity [23,46-50]. However, laboratory measurements of ternary amine-enhanced nucleation are still so complicated that experimental information on nucleation rates is still far from complete, despite the impressive progress achieved in instrumentation over the last decades. For example, the agreement between two available experimental studies concerning the DMA-enhanced nucleation, those of Almeida et al. [23] and Yu et al. [46], are obviously far from perfect. In particular, while Almeida et al. [23] claim that "addition of only 5 pptv DMA enhances the nucleation rate of sulphuric acid particles by more than six orders of magnitude", Yu et al. [46] found a much smaller (a factor of 4 to 100) enhancement due to DMA at much larger [DMA] varying from of $\sim 700 \mathrm{pptv}$ to $4000 \mathrm{pptv}$. Yu et al. [46] have also found that addition of ammonia to the ternary sulfuric acid-water-DMA mixture can enhance nucleation rates by a factor of $\sim 10$. More recently, Berndt et al. [48] have carried out a comprehensive experimental study of several amines, including the DMA. All amines showed a strong nucleation-enhancing effect. They also found that the enhancement factors decrease in the order trimethylamine>dimethylamine and aniline. While all the common alphatic amines, such as MA, DMA, and TMA, have high affinity for $\mathrm{H}_{2} \mathrm{SO}_{4}-\mathrm{H}_{2} \mathrm{O}$ clusters and can thus stabilize them [19-22], the recent laboratory studies suggest $\mathrm{NH}_{3}<\mathrm{MA}<\mathrm{TMA} \lesssim \mathrm{DMA}$ in stabilizing sulfuric acid clusters [46,50]. This indicates that MA, whose background atmospheric concentrations are estimated to be smaller than those of DMA and TMA [34], can be used to estimate the lower limit of the impact of aliphatic amines on atmospheric nucleation.

In this paper, the lower limit of the impact of methylamines on nucleation in the Earth's atmosphere has been studied using the DFT PW91PW91/6-311++G (3df, 3pd) method. The formation of stable sulfuric acid monomers and dimers containing one and two MA molecules and up to five $\mathrm{H}_{2} \mathrm{O}$ molecules has been investigated, new thermochemical data have been reported and atmospheric implications of the obtained results have been discussed. We have carried out a detailed thermochemical analysis, the comparison of the enhancement due to MA and ammonia and search for favorable conditions, at which MA could considerably enhance the formation of new particles in the Earth's atmosphere.

\section{Methods}

In the present study, we have employed the conventional DFT PW91PW91/6-311++G (3df, 3pd) method. Initial/guess geometries have been optimized using the PM3 method first. Then, the most stable isomers located within $10 \mathrm{kcal} / \mathrm{mol}$ of the intermediate global minima at the PM3 level have been treated using the PW91PW91/6-31+G* method. Finally, the most stable isomers located within $3 \mathrm{kcal} / \mathrm{mol}$ of the intermediate global minima at PW91PW91/6-31+G* level have been optimized using the PW91PW91/6-311++G (3df, 3pd) method. The number of initial/guess geometries ranged from fewer than 10-15 in the case when $\mathrm{H}_{2} \mathrm{O}$ was not involved in cluster formation to more than 70 in the case when high $(\mathrm{k}>3)$ hydrates are formed. The performance of the PW91PW91/6-311++G (3df, 3pd) method has been systematically validated against experimental Gibbs free energies for clusters relevant to the Earth's atmosphere $[6,22,25,52-54]$ and has shown a very good agreement with all the currently 
available experiments and higher level ab initio studies [55-59]. The method has been successfully applied to a wide range of nucleation problems including the classical problem Wilson's of the sign preference [52], stability and dipole moment of sulfuric acid hydrates, atmospheric nucleation of $\mathrm{H}_{2} \mathrm{SO}_{4}$ $\mathrm{H}_{2} \mathrm{O}$ ions of different sign and composition [53], temperature and concentration dependencies of the $\mathrm{H}_{2} \mathrm{O}$ nucleation rates [54], and impact of ammonia and organic acids on the stability of neutral and charged binary $\mathrm{H}_{2} \mathrm{SO}_{4}-\mathrm{H}_{2} \mathrm{O}$ clusters. A more detailed comparison of PW91PW91 free energies with other $a b$ initio and experimental data can be found in the Supplementary Information. The calculations of cluster distributions have been carried out using the standard chemical kinetics equations (see references [21,58] for details). The present work extends the recent study of Bustos et al. [58], where $\left(\mathrm{H}_{2} \mathrm{SO}_{4}\right)_{1}\left(\mathrm{CH}_{3} \mathrm{NH}_{2}\right)_{1}\left(\mathrm{H}_{2} \mathrm{O}\right)_{0-5}$ clusters have been studied using RI-MP2/CBS method. Compared to [58], the present study covers much larger range of cluster sizes and compositions. In particular, the present study covers the whole range of $\left(\mathrm{H}_{2} \mathrm{SO}_{4}\right)_{0-2}\left(\mathrm{CH}_{3} \mathrm{NH}_{2}\right)_{0-2}\left(\mathrm{H}_{2} \mathrm{O}\right)_{0-5}$ clusters. Calculations have been carried out using the Gaussian 09 suite of programs [60].

\section{Results and Discussion}

\subsection{Equilibrium Geometries and Cluster Properties}

Figure 1 present examples of the equilibrium geometries of the most stable isomers of $\left(\mathrm{H}_{2} \mathrm{SO}_{4}\right)_{\mathrm{n}}\left(\mathrm{CH}_{3} \mathrm{NH}_{2}\right)_{\mathrm{m}}\left(\mathrm{H}_{2} \mathrm{O}\right)_{\mathrm{k}}$ The formation of $\left(\mathrm{H}_{2} \mathrm{SO}_{4}\right)_{n}\left(\mathrm{CH}_{3} \mathrm{NH}_{2}\right)_{\mathrm{m}}\left(\mathrm{H}_{2} \mathrm{O}\right)_{\mathrm{k}}$ clusters is often accompanied by proton transfer.

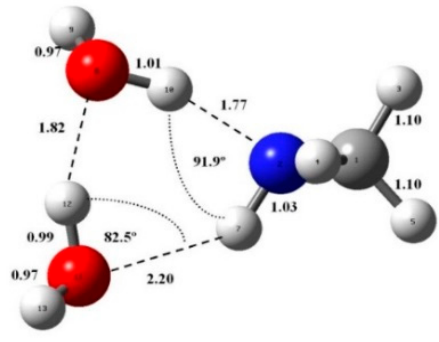

(a)

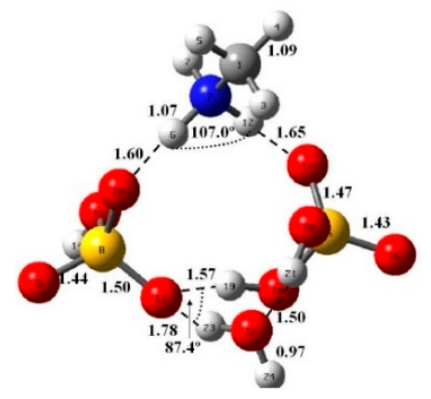

(d)

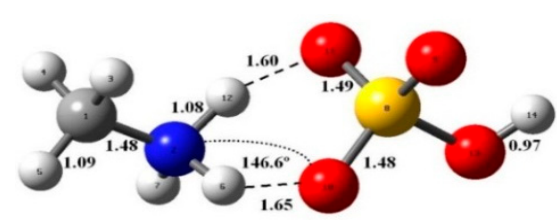

(b)

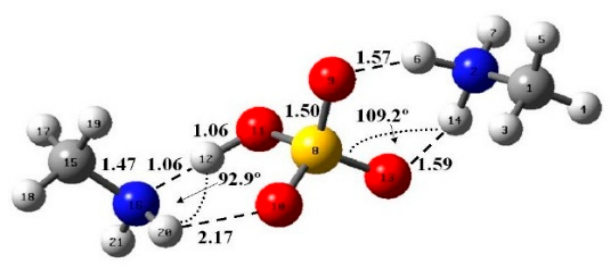

(e)

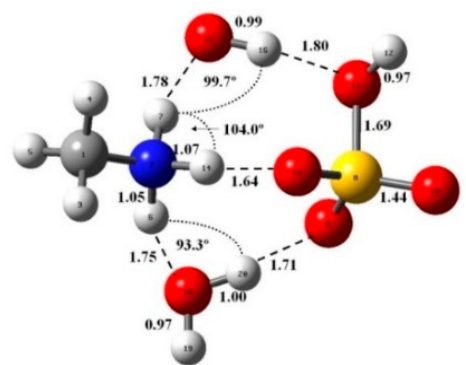

(c)

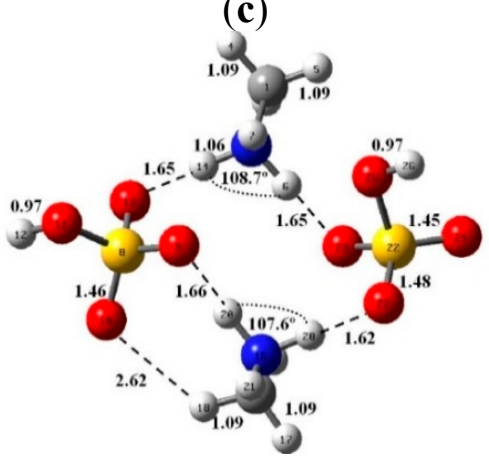

(f)

Figure 1. Equilibrium geometries of the most stable isomers of $\left(\mathrm{CH}_{3} \mathrm{NH}_{2}\right)\left(\mathrm{H}_{2} \mathrm{O}\right)_{2}$ (a); $\left.\left(\mathrm{H}_{2} \mathrm{SO}_{4}\right)\left(\mathrm{CH}_{3} \mathrm{NH}_{2}\right) \quad(\mathbf{b}),\left(\mathrm{H}_{2} \mathrm{SO}_{4}\right)\left(\mathrm{CH}_{3} \mathrm{NH}_{2}\right) \mathrm{H}_{2} \mathrm{O}\right)_{2} \quad(\mathbf{c}),\left(\mathrm{H}_{2} \mathrm{SO}_{4}\right)_{2}\left(\mathrm{CH}_{3} \mathrm{NH}_{2}\right)_{1}\left(\mathrm{H}_{2} \mathrm{O}\right)_{1} \quad$ (d), $\left(\mathrm{H}_{2} \mathrm{SO}_{4}\right)\left(\mathrm{CH}_{3} \mathrm{NH}_{2}\right)_{2}$ (e) and $\left(\mathrm{H}_{2} \mathrm{SO}_{4}\right)_{2} \quad\left(\mathrm{CH}_{3} \mathrm{NH}_{2}\right)_{2}$ (f) obtained at PW91PW91/ $6-311++\mathrm{G}(3 \mathrm{df}, 3 \mathrm{pd})$ level of theory. 
For example, in the cases of $\left(\mathrm{H}_{2} \mathrm{SO}_{4}\right)_{1}\left(\mathrm{CH}_{3} \mathrm{NH}_{2}\right)_{1}\left(\mathrm{H}_{2} \mathrm{O}\right)_{2}$ and $\left(\mathrm{H}_{2} \mathrm{SO}_{4}\right)_{2}\left(\mathrm{CH}_{3} \mathrm{NH}_{2}\right)_{2}$ clusters shown in Figure 1, the structural formulas of the most stable isomers of $\left(\mathrm{H}_{2} \mathrm{SO}_{4}\right)_{1}\left(\mathrm{CH}_{3} \mathrm{NH}_{2}\right)_{1}\left(\mathrm{H}_{2} \mathrm{O}\right)_{2}$ and $\left(\mathrm{H}_{2} \mathrm{SO}_{4}\right)_{2}\left(\mathrm{CH}_{3} \mathrm{NH}_{2}\right)_{2}$ are $\left(\mathrm{HSO}_{4}^{-}\right)\left(\mathrm{CH}_{3} \mathrm{NH}_{3}\right)^{+}\left(\mathrm{H}_{2} \mathrm{O}\right)_{2}$ and $\left(\mathrm{HSO}_{4}{ }^{-}\right)_{2}\left(\mathrm{CH}_{3} \mathrm{NH}_{3}{ }^{+}\right)_{2}$, respectively. Typically, $\mathrm{OH}$ bond lengths and $\mathrm{O}-\mathrm{O}$ distances in $\left(\mathrm{H}_{2} \mathrm{SO}_{4}\right)_{n}\left(\mathrm{CH}_{3} \mathrm{NH}_{2}\right)_{\mathrm{m}}\left(\mathrm{H}_{2} \mathrm{O}\right)_{\mathrm{k}}$ are a bit shorter than those in $\left(\mathrm{H}_{2} \mathrm{SO}_{4}\right)_{n}\left(\mathrm{NH}_{3}\right)_{\mathrm{m}}\left(\mathrm{H}_{2} \mathrm{O}\right)_{\mathrm{k}}$, being a sign of enhanced thermodynamic stability of $\left(\mathrm{H}_{2} \mathrm{SO}_{4}\right)_{n}\left(\mathrm{CH}_{3} \mathrm{NH}_{2}\right)_{\mathrm{m}}$ $\left(\mathrm{H}_{2} \mathrm{O}\right)_{\mathrm{k}}$ compared to $\left(\mathrm{H}_{2} \mathrm{SO}_{4}\right)_{\mathrm{n}}\left(\mathrm{NH}_{3}\right)_{\mathrm{m}}\left(\mathrm{H}_{2} \mathrm{O}\right)_{\mathrm{k}}$. In the Supplementary Materials, an interested reader can find the complete data set (over 180 equilibrium geometries in total) for the Cartesian geometries of global and local minima located within $\sim 3 \mathrm{kcal} / \mathrm{mol}$ of global minima, which cover the whole range of $\left(\mathrm{H}_{2} \mathrm{SO}_{4}\right)_{\mathrm{n}}\left(\mathrm{CH}_{3} \mathrm{NH}_{2}\right)_{\mathrm{m}}\left(\mathrm{H}_{2} \mathrm{O}\right)_{\mathrm{k}}$, from $\left(\mathrm{CH}_{3} \mathrm{NH}_{2}\right)$ molecules and $\left(\mathrm{CH}_{3} \mathrm{NH}_{2}\right)$ hydrates to $\left(\mathrm{H}_{2} \mathrm{SO}_{4}\right)_{2}$ $\left(\mathrm{CH}_{3} \mathrm{NH}_{2}\right)_{2}\left(\mathrm{H}_{2} \mathrm{O}\right)_{5}$, respectively. The corresponding data sets for $\left(\mathrm{H}_{2} \mathrm{SO}_{4}\right)_{n}\left(\mathrm{NH}_{3}\right)_{\mathrm{m}}\left(\mathrm{H}_{2} \mathrm{O}\right)_{k}$ and $\left.\left(\mathrm{H}_{2} \mathrm{SO}_{4}\right)_{\mathrm{n}}\left(\mathrm{CH}_{3}\right)_{2} \mathrm{NH}\right)_{\mathrm{m}}\left(\mathrm{H}_{2} \mathrm{O}\right)_{\mathrm{k}}$ are available in [26,61] and [22], respectively. Intrinsic statistical and entropic effects related to the difference between the Boltzmann-Gibbs average over the isomer mixture of a given composition and the energy of the global minimum of the same composition and to the difference in entropies between the global and minima do not exceed $0.1 \mathrm{kcal} \mathrm{mol}^{-1}$ in all the cases studied and can be neglected.

\subsection{New Thermochemical Data and Their Analysis}

\subsubsection{Hydration}

Table 1 presents enthalpies, entropies and Gibbs free energy changes associated with hydration, the formation of a shell consisting of $\mathrm{H}_{2} \mathrm{O}$ around $\left(\mathrm{H}_{2} \mathrm{SO}_{4}\right)_{\mathrm{m}}\left(\mathrm{CH}_{3} \mathrm{NH}_{2}\right)_{n}$ core. As seen from Table 1, the hydration of $\mathrm{CH}_{3} \mathrm{NH}_{2}$ is weak and, thus, one cannot expect $\left(\mathrm{CH}_{3} \mathrm{NH}_{2}\right)\left(\mathrm{H}_{2} \mathrm{O}\right)_{n}$ to be stable under atmospheric conditions. The hydration free energies of $\mathrm{CH}_{3} \mathrm{NH}_{2}$ vary from -1.46 to $1.7 \mathrm{kcal} \mathrm{mol}^{-1}$ that gives us a clear indication of instability of $\left(\mathrm{CH}_{3} \mathrm{NH}_{2}\right)\left(\mathrm{H}_{2} \mathrm{O}\right)_{n}$ complexes under atmospheric conditions. Bonding of $\mathrm{CH}_{3} \mathrm{NH}_{2}$ with $\mathrm{H}_{2} \mathrm{SO}_{4}$ significantly increases the hydration strength and leads to the formation of stable hydrated clusters. In particular, hydration of three $\left(\mathrm{H}_{2} \mathrm{SO}_{4}\right)\left(\mathrm{CH}_{3} \mathrm{NH}_{2}\right),\left(\mathrm{H}_{2} \mathrm{SO}_{4}\right)_{2}\left(\mathrm{CH}_{3} \mathrm{NH}_{2}\right)_{1}$ and $\left(\mathrm{H}_{2} \mathrm{SO}_{4}\right)_{2}\left(\mathrm{CH}_{3} \mathrm{NH}_{2}\right)_{2}$ out of four $\left(\mathrm{H}_{2} \mathrm{SO}_{4}\right)_{\mathrm{m}}\left(\mathrm{CH}_{3} \mathrm{NH}_{2}\right)_{\mathrm{n}}$ cores investigated in the present study is strong.

Table 1. Changes in enthalpies $\Delta \mathrm{H}\left(\mathrm{kcal} \mathrm{mol}^{-1}\right)$, entropies $\Delta \mathrm{S}\left(\mathrm{cal} \mathrm{mol}{ }^{-1} \mathrm{~K}^{-1}\right)$, and Gibbs free energy $\Delta \mathrm{G} \quad\left(\mathrm{kcal} \mathrm{mol}^{-1}\right)$ associated with hydration of nucleating $\left(\mathrm{H}_{2} \mathrm{SO}_{4}\right)_{\mathrm{n}}\left(\mathrm{CH}_{3} \mathrm{NH}_{2}\right)_{\mathrm{m}}\left(\mathrm{H}_{2} \mathrm{O}\right)_{\mathrm{k}}$ clusters at $298.15 \mathrm{~K}$ and pressure of $101.3 \mathrm{KPa}$. Subscript BA refers to the best ab initio RI-MP2/CBS study of Bustos et al. [58].

\begin{tabular}{lrrrr}
\hline \multicolumn{1}{c}{ Reaction } & $\Delta \mathbf{H}$ & $\Delta \mathbf{S}$ & $\Delta \mathbf{G}$ & $\Delta \mathbf{G}_{\mathbf{B A}}$ \\
\hline$\left(\mathrm{CH}_{3} \mathrm{NH}_{2}\right)+\left(\mathrm{H}_{2} \mathrm{O}\right)<=>\left(\mathrm{CH}_{3} \mathrm{NH}_{2}\right)\left(\mathrm{H}_{2} \mathrm{O}\right)$ & -7.73 & -22.25 & -1.10 & \\
$\left(\mathrm{CH}_{3} \mathrm{NH}_{2}\right)\left(\mathrm{H}_{2} \mathrm{O}\right)+\left(\mathrm{H}_{2} \mathrm{O}\right)<=>\left(\mathrm{CH}_{3} \mathrm{NH}_{2}\right)\left(\mathrm{H}_{2} \mathrm{O}\right)_{2}$ & -8.70 & -34.40 & 1.55 & \\
$\left(\mathrm{CH}_{3} \mathrm{NH}_{2}\right)\left(\mathrm{H}_{2} \mathrm{O}\right)_{2}+\left(\mathrm{H}_{2} \mathrm{O}\right)<=>\left(\mathrm{CH}_{3} \mathrm{NH}_{2}\right)\left(\mathrm{H}_{2} \mathrm{O}\right)_{3}$ & -11.21 & -32.72 & -1.46 & \\
$\left(\mathrm{CH}_{3} \mathrm{NH}\right)\left(\mathrm{H}_{2} \mathrm{O}\right)_{3}+\left(\mathrm{H}_{2} \mathrm{O}\right)<=>\left(\mathrm{CH}_{3} \mathrm{NH}_{\left(\mathrm{H}_{2} \mathrm{O}\right)_{4}}\right.$ & -9.10 & -29.31 & -0.36 & \\
$\left(\mathrm{CH}_{3} \mathrm{NH}_{2}\right)\left(\mathrm{H}_{2} \mathrm{O}\right)_{4}+\left(\mathrm{H}_{2} \mathrm{O}\right)<=>\left(\mathrm{CH}_{3} \mathrm{NH}_{2}\right)\left(\mathrm{H}_{2} \mathrm{O}\right)_{5}$ & -7.36 & -30.37 & 1.70 & \\
$\left(\mathrm{H}_{2} \mathrm{SO}_{4}\right)\left(\mathrm{CH}_{3} \mathrm{NH}_{2}\right)+\left(\mathrm{H}_{2} \mathrm{O}\right)<=>\left(\mathrm{H}_{2} \mathrm{SO}_{4}\right)\left(\mathrm{CH}_{3} \mathrm{NH}_{2}\right)\left(\mathrm{H}_{2} \mathrm{O}\right)$ & -13.02 & -32.49 & -3.33 & -3.53 \\
$\left(\mathrm{H}_{2} \mathrm{SO}_{4}\right)\left(\mathrm{CH}_{3} \mathrm{NH}_{2}\right)\left(\mathrm{H}_{2} \mathrm{O}\right)+\left(\mathrm{H}_{2} \mathrm{O}\right)<=>\left(\mathrm{H}_{2} \mathrm{SO}_{4}\right)\left(\mathrm{CH}_{3} \mathrm{NH}_{2}\right)\left(\mathrm{H}_{2} \mathrm{O}\right)_{2}$ & -13.85 & -34.66 & -3.52 & -3.95 \\
$\left(\mathrm{H}_{2} \mathrm{SO}_{4}\right)\left(\mathrm{CH}_{3} \mathrm{NH}_{2}\right)\left(\mathrm{H}_{2} \mathrm{O}\right)_{2}+\left(\mathrm{H}_{2} \mathrm{O}\right)<=>\left(\mathrm{H}_{2} \mathrm{SO}_{4}\right)\left(\mathrm{CH}_{3} \mathrm{NH}_{2}\right)\left(\mathrm{H}_{2} \mathrm{O}\right)_{3}$ & -10.97 & -29.73 & -2.11 & \\
\hline
\end{tabular}


Table 1. Cont.

\begin{tabular}{|c|c|c|c|c|}
\hline Reaction & $\Delta \mathbf{H}$ & $\Delta \mathbf{S}$ & $\Delta \mathbf{G}$ & $\Delta \mathbf{G}_{\mathrm{BA}}$ \\
\hline$\left(\mathrm{H}_{2} \mathrm{SO}_{4}\right)\left(\mathrm{CH}_{3} \mathrm{NH}_{2}\right)\left(\mathrm{H}_{2} \mathrm{O}\right)_{3}+\left(\mathrm{H}_{2} \mathrm{O}\right)<=\left(\mathrm{H}_{2} \mathrm{SO}_{4}\right)\left(\mathrm{CH}_{3} \mathrm{NH}_{2}\right)\left(\mathrm{H}_{2} \mathrm{O}\right)_{4}$ & -10.61 & -31.21 & -1.31 & \\
\hline$\left(\mathrm{H}_{2} \mathrm{SO}_{4}\right)\left(\mathrm{CH}_{3} \mathrm{NH}_{2}\right)\left(\mathrm{H}_{2} \mathrm{O}\right)_{4}+\left(\mathrm{H}_{2} \mathrm{O}\right)<=\left(\mathrm{H}_{2} \mathrm{SO}_{4}\right)\left(\mathrm{CH}_{3} \mathrm{NH}_{2}\right)\left(\mathrm{H}_{2} \mathrm{O}\right)_{5}$ & -11.84 & -39.55 & -0.05 & \\
\hline$\left(\mathrm{H}_{2} \mathrm{SO}_{4}\right)\left(\mathrm{CH}_{3} \mathrm{NH}_{2}\right)_{2}+\left(\mathrm{H}_{2} \mathrm{O}\right)<=\left(\mathrm{H}_{2} \mathrm{SO}_{4}\right)\left(\mathrm{CH}_{3} \mathrm{NH}_{2}\right)_{2}\left(\mathrm{H}_{2} \mathrm{O}\right)$ & -12.26 & -36.04 & -1.51 & \\
\hline$\left(\mathrm{H}_{2} \mathrm{SO}_{4}\right)\left(\mathrm{CH}_{3} \mathrm{NH}_{2}\right)_{2}\left(\mathrm{H}_{2} \mathrm{O}\right)+\left(\mathrm{H}_{2} \mathrm{O}\right)<=\left(\mathrm{H}_{2} \mathrm{SO}_{4}\right)\left(\mathrm{CH}_{3} \mathrm{NH}_{2}\right)_{2}\left(\mathrm{H}_{2} \mathrm{O}\right)_{2}$ & -11.73 & -35.65 & -1.10 & \\
\hline$\left(\mathrm{H}_{2} \mathrm{SO}_{4}\right)\left(\mathrm{CH}_{3} \mathrm{NH}_{2}\right)_{2}\left(\mathrm{H}_{2} \mathrm{O}\right)_{2}+\left(\mathrm{H}_{2} \mathrm{O}\right)<=>\left(\mathrm{H}_{2} \mathrm{SO}_{4}\right)\left(\mathrm{CH}_{3} \mathrm{NH}_{2}\right)_{2}\left(\mathrm{H}_{2} \mathrm{O}\right)_{3}$ & -13.30 & -35.31 & -2.77 & \\
\hline$\left(\mathrm{H}_{2} \mathrm{SO}_{4}\right)\left(\mathrm{CH}_{3} \mathrm{NH}_{2}\right)_{2}\left(\mathrm{H}_{2} \mathrm{O}\right)_{3}+\left(\mathrm{H}_{2} \mathrm{O}\right)<=\left(\mathrm{H}_{2} \mathrm{SO}_{4}\right)\left(\mathrm{CH}_{3} \mathrm{NH}_{2}\right)_{2}\left(\mathrm{H}_{2} \mathrm{O}\right)_{4}$ & -11.30 & -33.74 & -1.24 & \\
\hline$\left(\mathrm{H}_{2} \mathrm{SO}_{4}\right)_{2}\left(\mathrm{CH}_{3} \mathrm{NH}_{2}\right)+\left(\mathrm{H}_{2} \mathrm{O}\right)<=>\left(\mathrm{H}_{2} \mathrm{SO}_{4}\right)_{2}\left(\mathrm{CH}_{3} \mathrm{NH}_{2}\right)\left(\mathrm{H}_{2} \mathrm{O}\right)$ & -11.26 & -31.27 & -1.94 & \\
\hline$\left(\mathrm{H}_{2} \mathrm{SO}_{4}\right)_{2}\left(\mathrm{CH}_{3} \mathrm{NH}_{2}\right)\left(\mathrm{H}_{2} \mathrm{O}\right)+\left(\mathrm{H}_{2} \mathrm{O}\right)<=\left(\mathrm{H}_{2} \mathrm{SO}_{4}\right)_{2}\left(\mathrm{CH}_{3} \mathrm{NH}_{2}\right)\left(\mathrm{H}_{2} \mathrm{O}\right)_{2}$ & -13.49 & -34.61 & -3.17 & \\
\hline$\left(\mathrm{H}_{2} \mathrm{SO}_{4}\right)_{2}\left(\mathrm{CH}_{3} \mathrm{NH}_{2}\right)\left(\mathrm{H}_{2} \mathrm{O}\right)_{2}+\left(\mathrm{H}_{2} \mathrm{O}\right)<=>\left(\mathrm{H}_{2} \mathrm{SO}_{4}\right)_{2}\left(\mathrm{CH}_{3} \mathrm{NH}_{2}\right)\left(\mathrm{H}_{2} \mathrm{O}\right)_{3}$ & -11.99 & -31.32 & -2.65 & \\
\hline$\left(\mathrm{H}_{2} \mathrm{SO}_{4}\right)_{2}\left(\mathrm{CH}_{3} \mathrm{NH}_{2}\right)\left(\mathrm{H}_{2} \mathrm{O}\right)_{3}+\left(\mathrm{H}_{2} \mathrm{O}\right)<=\left(\mathrm{H}_{2} \mathrm{SO}_{4}\right)_{2}\left(\mathrm{CH}_{3} \mathrm{NH}_{2}\right)\left(\mathrm{H}_{2} \mathrm{O}\right)_{4}$ & -12.33 & -35.18 & -1.84 & \\
\hline$\left(\mathrm{H}_{2} \mathrm{SO}_{4}\right)_{2}\left(\mathrm{CH}_{3} \mathrm{NH}_{2}\right)\left(\mathrm{H}_{2} \mathrm{O}\right)_{4}+\left(\mathrm{H}_{2} \mathrm{O}\right)<=\left(\mathrm{H}_{2} \mathrm{SO}_{4}\right)_{2}\left(\mathrm{CH}_{3} \mathrm{NH}_{2}\right)\left(\mathrm{H}_{2} \mathrm{O}\right)_{5}$ & -10.82 & -30.46 & -1.74 & \\
\hline$\left(\mathrm{H}_{2} \mathrm{SO}_{4}\right)_{2}\left(\mathrm{CH}_{3} \mathrm{NH}_{2}\right)_{2}+\left(\mathrm{H}_{2} \mathrm{O}\right)<=\left(\mathrm{H}_{2} \mathrm{SO}_{4}\right)_{2}\left(\mathrm{CH}_{3} \mathrm{NH}_{2}\right)_{2}\left(\mathrm{H}_{2} \mathrm{O}\right)$ & -12.07 & -36.10 & -1.31 & \\
\hline$\left(\mathrm{H}_{2} \mathrm{SO}_{4}\right)_{2}\left(\mathrm{CH}_{3} \mathrm{NH}_{2}\right)_{2}\left(\mathrm{H}_{2} \mathrm{O}\right)+\left(\mathrm{H}_{2} \mathrm{O}\right)<=\left(\mathrm{H}_{2} \mathrm{SO}_{4}\right)_{2}\left(\mathrm{CH}_{3} \mathrm{NH}_{2}\right)_{2}\left(\mathrm{H}_{2} \mathrm{O}\right)_{2}$ & -11.62 & -29.53 & -2.82 & \\
\hline$\left(\mathrm{H}_{2} \mathrm{SO}_{4}\right)_{2}\left(\mathrm{CH}_{3} \mathrm{NH}_{2}\right)_{2}\left(\mathrm{H}_{2} \mathrm{O}\right)_{2}+\left(\mathrm{H}_{2} \mathrm{O}\right)<=>\left(\mathrm{H}_{2} \mathrm{SO}_{4}\right)_{2}\left(\mathrm{CH}_{3} \mathrm{NH}_{2}\right)_{2}\left(\mathrm{H}_{2} \mathrm{O}\right)_{3}$ & -14.55 & -32.83 & -4.76 & \\
\hline$\left(\mathrm{H}_{2} \mathrm{SO}_{4}\right)_{2}\left(\mathrm{CH}_{3} \mathrm{NH}_{2}\right)_{2}\left(\mathrm{H}_{2} \mathrm{O}\right)_{3}+\left(\mathrm{H}_{2} \mathrm{O}\right)<=\left(\mathrm{H}_{2} \mathrm{SO}_{4}\right)_{2}\left(\mathrm{CH}_{3} \mathrm{NH}_{2}\right)_{2}\left(\mathrm{H}_{2} \mathrm{O}\right)_{4}$ & -11.09 & -31.28 & -1.77 & \\
\hline$\left(\mathrm{H}_{2} \mathrm{SO}_{4}\right)_{2}\left(\mathrm{CH}_{3} \mathrm{NH}_{2}\right)_{2}\left(\mathrm{H}_{2} \mathrm{O}\right)_{4}+\left(\mathrm{H}_{2} \mathrm{O}\right)<=\left(\mathrm{H}_{2} \mathrm{SO}_{4}\right)_{2}\left(\mathrm{CH}_{3} \mathrm{NH}_{2}\right)_{2}\left(\mathrm{H}_{2} \mathrm{O}\right)_{5}$ & -11.25 & -33.56 & -1.24 & \\
\hline
\end{tabular}

The hydration of $\left(\mathrm{H}_{2} \mathrm{SO}_{4}\right)\left(\mathrm{CH}_{3} \mathrm{NH}_{2}\right)_{2}$ is much weaker than that of $\left(\left(\mathrm{H}_{2} \mathrm{SO}_{4}\right)\left(\mathrm{CH}_{3} \mathrm{NH}_{2}\right)\right.$, $\left(\mathrm{H}_{2} \mathrm{SO}_{4}\right)_{2}\left(\mathrm{CH}_{3} \mathrm{NH}_{2}\right)_{1}$ and $\left(\mathrm{H}_{2} \mathrm{SO}_{4}\right)_{2}\left(\mathrm{CH}_{3} \mathrm{NH}_{2}\right)_{2}$; however, it is still strong enough to impact the rates, at which $\left(\mathrm{H}_{2} \mathrm{SO}_{4}\right)\left(\mathrm{CH}_{3} \mathrm{NH}_{2}\right)_{2}$ are formed, especially at high $\mathrm{RH}$. The hydration of $\left(\mathrm{H}_{2} \mathrm{SO}_{4}\right)\left(\mathrm{CH}_{3} \mathrm{NH}_{2}\right)$ and $\left(\mathrm{H}_{2} \mathrm{SO}_{4}\right)\left(\mathrm{CH}_{3} \mathrm{NH}_{2}\right)\left(\mathrm{H}_{2} \mathrm{O}\right)$ obtained in the present study agrees well with the recent the higher level $a b$ initio RI-MP2/CBS study by Bustos et al. [58]

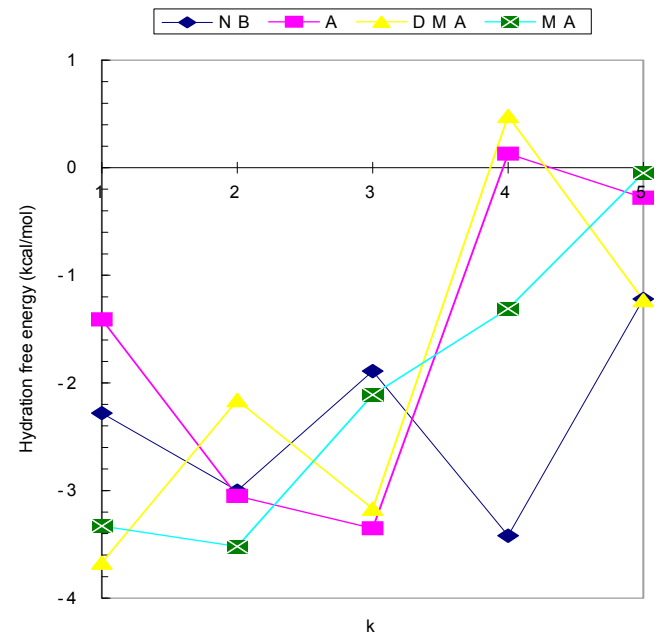

(a)

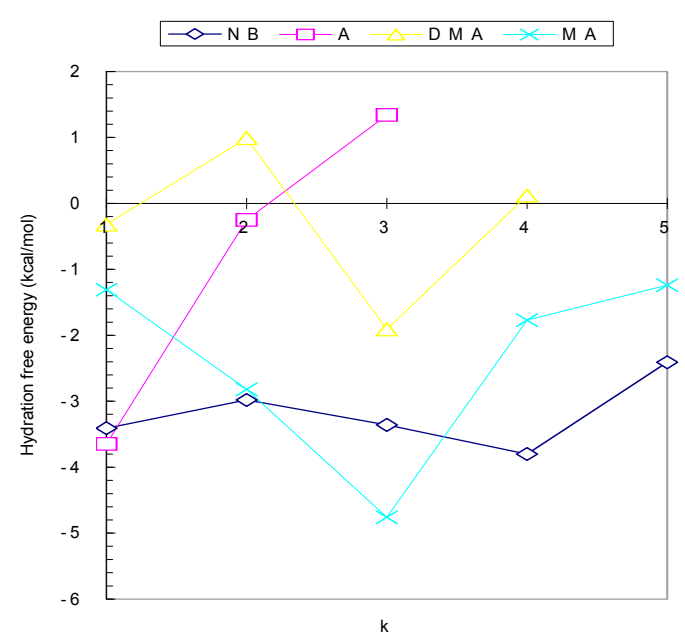

(b)

Figure 2. Standard conditions $(\mathrm{T}=298 \mathrm{~K}, \mathrm{P}=1013 \mathrm{KPa})$ hydration free energies $\left(\mathrm{kcal} \mathrm{mol}^{-1}\right)$ for $\left(\mathrm{H}_{2} \mathrm{SO}_{4}\right)_{1}(\text { Base })_{1}$ (a) and $\left(\mathrm{H}_{2} \mathrm{SO}_{4}\right)_{2}(\text { Base })_{2}$ (b) clusters. Abbreviations NB, A, DMA and MA refer to no base, ammonia, dimethylamine and methylamine, respectively. The data for ammonia and dimethylamine were adopted from [26,61] and [22], respectively. 
The comparison of hydration free energies for clusters containing monomers and dimers of $\mathrm{H}_{2} \mathrm{SO}_{4}$ and bases such as ammonia, DMA and MA shown in Figure 2 indicates that the hydration of the MA is stronger than that of other common atmospheric bases, $\mathrm{NH}_{3}$ and DMA. These considerations lead us to conclude that rates, at which $\left(\mathrm{H}_{2} \mathrm{SO}_{4}\right)_{\mathrm{m}}\left(\mathrm{CH}_{3} \mathrm{NH}_{2}\right)_{\mathrm{n}}\left(\mathrm{H}_{2} \mathrm{O}\right)_{\mathrm{k}}$ stable pre-nucleation clusters are formed, are definitely RH-dependent.

\subsubsection{Affinity of $\mathrm{H}_{2} \mathrm{SO}_{4}$ to Nucleating $\left(\mathrm{H}_{2} \mathrm{SO}_{4}\right)_{\mathrm{m}}\left(\mathrm{CH}_{3} \mathrm{NH}_{2}\right)_{n}\left(\mathrm{H}_{2} \mathrm{O}\right)_{k}$ Clusters}

Table 2 presents the standard conditions affinities of $\mathrm{H}_{2} \mathrm{SO}_{4}$ to nucleating $\left(\mathrm{H}_{2} \mathrm{SO}_{4}\right)_{n}\left(\mathrm{CH}_{3} \mathrm{NH}_{2}\right)_{\mathrm{m}}\left(\mathrm{H}_{2} \mathrm{O}\right)_{\mathrm{k}}$ clusters.

Table 2. Changes in enthalpies $\Delta \mathrm{H}\left(\mathrm{kcal} \mathrm{mol}^{-1}\right)$, entropies $\Delta \mathrm{S}$ cal mol$\left.{ }^{-1} \mathrm{~K}^{-1}\right)$, and Gibbs free energy $\quad \Delta \mathrm{G} \quad\left(\mathrm{kcal} \mathrm{mol}^{-1}\right)$ describing the affinity of $\mathrm{H}_{2} \mathrm{SO}_{4}$ to nucleating $\left(\mathrm{H}_{2} \mathrm{SO}_{4}\right)_{\mathrm{n}}\left(\mathrm{CH}_{3} \mathrm{NH}_{2}\right)_{\mathrm{m}}\left(\mathrm{H}_{2} \mathrm{O}\right)_{\mathrm{k}}$ clusters at $298.15 \mathrm{~K}$ and pressure of $101.3 \mathrm{KPa}$. Subscript BA refers to the best ab initio RI-MP2/CBS study by Bustos et al. [58].

\begin{tabular}{|c|c|c|c|}
\hline Reaction & $\Delta \mathbf{H}$ & $\Delta \mathbf{S}$ & $\Delta \mathbf{G}$ \\
\hline$\left(\mathrm{CH}_{3} \mathrm{NH}_{2}\right)+\left(\mathrm{H}_{2} \mathrm{SO}_{4}\right)<=\left(\mathrm{H}_{2} \mathrm{SO}_{4}\right)\left(\mathrm{CH}_{3} \mathrm{NH}_{2}\right)$ & -20.40 & -31.42 & $-11.03(-11.61)_{\mathrm{BA}}$ \\
\hline$\left(\mathrm{CH}_{3} \mathrm{NH}_{2}\right)\left(\mathrm{H}_{2} \mathrm{O}\right)+\left(\mathrm{H}_{2} \mathrm{SO}_{4}\right)<=\left(\mathrm{H}_{2} \mathrm{SO}_{4}\right)\left(\mathrm{CH}_{3} \mathrm{NH}_{2}\right)\left(\mathrm{H}_{2} \mathrm{O}\right)$ & -25.69 & -41.67 & -13.26 \\
\hline$\left(\mathrm{CH}_{3} \mathrm{NH}_{2}\right)\left(\mathrm{H}_{2} \mathrm{O}\right)_{2}+\left(\mathrm{H}_{2} \mathrm{SO}_{4}\right)<=\left(\mathrm{H}_{2} \mathrm{SO}_{4}\right)\left(\mathrm{CH}_{3} \mathrm{NH}_{2}\right)\left(\mathrm{H}_{2} \mathrm{O}\right)_{2}$ & -30.83 & -41.93 & -18.33 \\
\hline$\left(\mathrm{CH}_{3} \mathrm{NH}_{2}\right)\left(\mathrm{H}_{2} \mathrm{O}\right)_{3}+\left(\mathrm{H}_{2} \mathrm{SO}_{4}\right) \Leftrightarrow>\left(\mathrm{H}_{2} \mathrm{SO}_{4}\right)\left(\mathrm{CH}_{3} \mathrm{NH}_{2}\right)\left(\mathrm{H}_{2} \mathrm{O}\right)_{3}$ & -30.59 & -38.95 & -18.98 \\
\hline$\left(\mathrm{CH}_{3} \mathrm{NH}_{2}\right)\left(\mathrm{H}_{2} \mathrm{O}\right)_{4}+\left(\mathrm{H}_{2} \mathrm{SO}_{4}\right) \Leftrightarrow>\left(\mathrm{H}_{2} \mathrm{SO}_{4}\right)\left(\mathrm{CH}_{3} \mathrm{NH}_{2}\right)\left(\mathrm{H}_{2} \mathrm{O}\right)_{4}$ & -32.11 & -40.85 & -19.93 \\
\hline$\left(\mathrm{CH}_{3} \mathrm{NH}_{2}\right)\left(\mathrm{H}_{2} \mathrm{O}\right)_{5}+\left(\mathrm{H}_{2} \mathrm{SO}_{4}\right)<=\left(\mathrm{H}_{2} \mathrm{SO}_{4}\right)\left(\mathrm{CH}_{3} \mathrm{NH}_{2}\right)\left(\mathrm{H}_{2} \mathrm{O}\right)_{5}$ & -36.60 & -50.04 & -21.68 \\
\hline$\left(\mathrm{H}_{2} \mathrm{SO}_{4}\right)\left(\mathrm{CH}_{3} \mathrm{NH}_{2}\right)+\left(\mathrm{H}_{2} \mathrm{SO}_{4}\right)<=\left(\mathrm{H}_{2} \mathrm{SO}_{4}\right)_{2}\left(\mathrm{CH}_{3} \mathrm{NH}_{2}\right)$ & -27.42 & -44.17 & -14.25 \\
\hline$\left(\mathrm{H}_{2} \mathrm{SO}_{4}\right)\left(\mathrm{CH}_{3} \mathrm{NH}_{2}\right)\left(\mathrm{H}_{2} \mathrm{O}\right)+\left(\mathrm{H}_{2} \mathrm{SO}_{4}\right) \Leftrightarrow>\left(\mathrm{H}_{2} \mathrm{SO}_{4}\right)_{2}\left(\mathrm{CH}_{3} \mathrm{NH}_{2}\right)\left(\mathrm{H}_{2} \mathrm{O}\right)$ & -25.66 & -42.94 & -12.86 \\
\hline$\left(\mathrm{H}_{2} \mathrm{SO}_{4}\right)\left(\mathrm{CH}_{3} \mathrm{NH}_{2}\right)\left(\mathrm{H}_{2} \mathrm{O}\right)_{2}+\left(\mathrm{H}_{2} \mathrm{SO}_{4}\right) \Leftrightarrow=\left(\mathrm{H}_{2} \mathrm{SO}_{4}\right)_{2}\left(\mathrm{CH}_{3} \mathrm{NH}_{2}\right)\left(\mathrm{H}_{2} \mathrm{O}\right)_{2}$ & -25.30 & -42.90 & -12.51 \\
\hline$\left(\mathrm{H}_{2} \mathrm{SO}_{4}\right)\left(\mathrm{CH}_{3} \mathrm{NH}_{2}\right)\left(\mathrm{H}_{2} \mathrm{O}\right)_{3}+\left(\mathrm{H}_{2} \mathrm{SO}_{4}\right)<=\left(\mathrm{H}_{2} \mathrm{SO} 4\right)_{2}\left(\mathrm{CH}_{3} \mathrm{NH}_{2}\right)\left(\mathrm{H}_{2} \mathrm{O}\right)_{3}$ & -26.31 & -44.49 & -13.05 \\
\hline$\left(\mathrm{H}_{2} \mathrm{SO}_{4}\right)\left(\mathrm{CH}_{3} \mathrm{NH}_{2}\right)\left(\mathrm{H}_{2} \mathrm{O}\right)_{4}+\left(\mathrm{H}_{2} \mathrm{SO}_{4}\right)<=\left(\mathrm{H}_{2} \mathrm{SO}_{4}\right)_{2}\left(\mathrm{CH}_{3} \mathrm{NH}_{2}\right)\left(\mathrm{H}_{2} \mathrm{O}\right)_{4}$ & -28.03 & -48.46 & -13.58 \\
\hline$\left(\mathrm{CH}_{3} \mathrm{NH}_{2}\right)_{2}+\left(\mathrm{H}_{2} \mathrm{SO}_{4}\right)<=>\left(\mathrm{H}_{2} \mathrm{SO}_{4}\right)\left(\mathrm{CH}_{3} \mathrm{NH}_{2}\right)_{2}$ & -31.93 & -42.75 & -19.18 \\
\hline$\left(\mathrm{CH}_{3} \mathrm{NH}_{2}\right)_{2}\left(\mathrm{H}_{2} \mathrm{O}\right)+\left(\mathrm{H}_{2} \mathrm{SO}_{4}\right) \Leftrightarrow>\left(\mathrm{H}_{2} \mathrm{SO}_{4}\right)\left(\mathrm{CH}_{3} \mathrm{NH}_{2}\right)_{2}\left(\mathrm{H}_{2} \mathrm{O}\right)$ & -33.74 & -45.36 & -20.22 \\
\hline$\left(\mathrm{CH}_{3} \mathrm{NH}_{2}\right)_{2}\left(\mathrm{H}_{2} \mathrm{O}\right)_{2}+\left(\mathrm{H}_{2} \mathrm{SO}_{4}\right)<=\left(\mathrm{H}_{2} \mathrm{SO}_{4}\right)\left(\mathrm{CH}_{3} \mathrm{NH}_{2}\right)_{2}\left(\mathrm{H}_{2} \mathrm{O}\right)_{2}$ & -33.81 & -49.43 & -19.07 \\
\hline$\left(\mathrm{CH}_{3} \mathrm{NH}_{2}\right)_{2}\left(\mathrm{H}_{2} \mathrm{O}\right)_{3}+\left(\mathrm{H}_{2} \mathrm{SO}_{4}\right)<=\left(\mathrm{H}_{2} \mathrm{SO}_{4}\right)\left(\mathrm{CH}_{3} \mathrm{NH}_{2}\right)_{2}\left(\mathrm{H}_{2} \mathrm{O}\right)_{3}$ & -38.21 & -54.86 & -21.85 \\
\hline$\left(\mathrm{CH}_{3} \mathrm{NH}_{2}\right)_{2}\left(\mathrm{H}_{2} \mathrm{O}\right)_{4}+\left(\mathrm{H}_{2} \mathrm{SO}_{4}\right)<=\left(\mathrm{H}_{2} \mathrm{SO}_{4}\right)\left(\mathrm{CH}_{3} \mathrm{NH}_{2}\right)_{2}\left(\mathrm{H}_{2} \mathrm{O}\right)_{4}$ & -42.26 & -60.50 & -24.22 \\
\hline$\left(\mathrm{H}_{2} \mathrm{SO}_{4}\right)\left(\mathrm{CH}_{3} \mathrm{NH}_{2}\right)_{2}+\left(\mathrm{H}_{2} \mathrm{SO}_{4}\right)<=\left(\mathrm{H}_{2} \mathrm{SO}_{4}\right)_{2}\left(\mathrm{CH}_{3} \mathrm{NH}_{2}\right)_{2}$ & -31.08 & -40.62 & -18.97 \\
\hline$\left(\mathrm{H}_{2} \mathrm{SO}_{4}\right)\left(\mathrm{CH}_{3} \mathrm{NH}_{2}\right)_{2}\left(\mathrm{H}_{2} \mathrm{O}\right)+\left(\mathrm{H}_{2} \mathrm{SO}_{4}\right) \Leftrightarrow=\left(\mathrm{H}_{2} \mathrm{SO}_{4}\right)_{2}\left(\mathrm{CH}_{3} \mathrm{NH}_{2}\right)_{2}\left(\mathrm{H}_{2} \mathrm{O}\right)$ & -30.89 & -40.68 & -18.76 \\
\hline$\left(\mathrm{H}_{2} \mathrm{SO}_{4}\right)\left(\mathrm{CH}_{3} \mathrm{NH}_{2}\right)_{2}\left(\mathrm{H}_{2} \mathrm{O}\right)_{2}+\left(\mathrm{H}_{2} \mathrm{SO}_{4}\right)<=\left(\mathrm{H}_{2} \mathrm{SO}_{4}\right)_{2}\left(\mathrm{CH}_{3 \mathrm{~N}} \mathrm{H}_{2}\right)_{2}\left(\mathrm{H}_{2} \mathrm{O}\right)_{2}$ & -30.78 & -34.57 & -20.48 \\
\hline$\left(\mathrm{H}_{2} \mathrm{SO}_{4}\right)\left(\mathrm{CH}_{3} \mathrm{NH}_{2}\right)_{2}\left(\mathrm{H}_{2} \mathrm{O}\right)_{3}+\left(\mathrm{H}_{2} \mathrm{SO}_{4}\right)<=\left(\mathrm{H}_{2} \mathrm{SO}_{4}\right)_{2}\left(\mathrm{CH}_{3} \mathrm{NH}_{2}\right)_{2}\left(\mathrm{H}_{2} \mathrm{O}\right)_{3}$ & -32.03 & -32.09 & -22.46 \\
\hline$\left(\mathrm{H}_{2} \mathrm{SO}_{4}\right)\left(\mathrm{CH}_{3} \mathrm{NH}_{2}\right)_{2}\left(\mathrm{H}_{2} \mathrm{O}\right)_{4}+\left(\mathrm{H}_{2} \mathrm{SO}_{4}\right)<=>\left(\mathrm{H}_{2} \mathrm{SO}_{4}\right)_{2}\left(\mathrm{CH}_{3} \mathrm{NH}_{2}\right)_{2}\left(\mathrm{H}_{2} \mathrm{O}\right)_{4}$ & -31.83 & -29.62 & -22.99 \\
\hline
\end{tabular}

The comparison of the new thermodynamic data summarized in Table 2 with data for $\left(\mathrm{H}_{2} \mathrm{SO}_{4}\right)_{n}\left(\mathrm{NH}_{3}\right)_{\mathrm{m}}\left(\mathrm{H}_{2} \mathrm{O}\right)_{\mathrm{k}}$ [47] shows clearly that the $\left(\mathrm{H}_{2} \mathrm{SO}_{4}\right)_{\mathrm{n}}\left(\mathrm{CH}_{3} \mathrm{NH}_{2}\right)_{\mathrm{m}}\left(\mathrm{H}_{2} \mathrm{O}\right)_{\mathrm{k}}$ clusters are much more stable than the corresponding $\left(\mathrm{H}_{2} \mathrm{SO}_{4}\right)_{\mathrm{n}}\left(\mathrm{NH}_{3}\right)_{\mathrm{m}}\left(\mathrm{H}_{2} \mathrm{O}\right)_{\mathrm{k}}$ ones. This is by no means surprising because MA is a much stronger base than $\mathrm{NH}_{3}$. The $\mathrm{H}_{2} \mathrm{SO}_{4}$ affinity to nucleating $\left(\mathrm{H}_{2} \mathrm{SO}_{4}\right)_{n}\left(\mathrm{CH}_{3} \mathrm{NH}_{2}\right)_{\mathrm{m}}\left(\mathrm{H}_{2} \mathrm{O}\right)_{\mathrm{k}}$ clusters tends to grow with molar fraction of the base $\left(\mathrm{CH}_{3} \mathrm{NH}_{2}\right)$ and varies with the hydration number. This pattern is nearly identical to that of the $\mathrm{H}_{2} \mathrm{SO}_{4}$ affinity to $\left(\mathrm{H}_{2} \mathrm{SO}_{4}\right)_{n}\left(\left(\mathrm{CH}_{3}\right)_{2} \mathrm{NH}\right)_{\mathrm{m}}\left(\mathrm{H}_{2} \mathrm{O}\right)_{k}$ and $\left(\mathrm{H}_{2} \mathrm{SO}_{4}\right) \mathrm{n}\left(\mathrm{NH}_{3}\right)_{\mathrm{m}}\left(\mathrm{H}_{2} \mathrm{O}\right)_{\mathrm{k}}$. 
As seen from Figure 3, which presents the comparison of standard conditions affinities of $\mathrm{H}_{2} \mathrm{SO}_{4}$ to nucleating $\left(\mathrm{H}_{2} \mathrm{SO}_{4}\right)_{\mathrm{n}}\left(\mathrm{CH}_{3} \mathrm{NH}_{2}\right)_{\mathrm{m}}\left(\mathrm{H}_{2} \mathrm{O}\right)_{\mathrm{k}}$ and $\left(\mathrm{H}_{2} \mathrm{SO}_{4}\right)_{\mathrm{n}}\left(\mathrm{CH}_{3} \mathrm{NH}_{2}\right)_{\mathrm{m}}\left(\mathrm{H}_{2} \mathrm{O}\right)_{\mathrm{k}}$ clusters, the affinities of $\mathrm{H}_{2} \mathrm{SO}_{4}$, the key atmospheric nucleation precursor to nucleating $\left(\mathrm{H}_{2} \mathrm{SO}_{4}\right)_{n}\left(\mathrm{CH}_{3} \mathrm{NH}_{2}\right)_{\mathrm{m}}\left(\mathrm{H}_{2} \mathrm{O}\right)_{\mathrm{k}}$ and $\left(\mathrm{H}_{2} \mathrm{SO}_{4}\right)_{\mathrm{m}}\left(\left(\mathrm{CH}_{3}\right)_{2} \mathrm{NH}\right)_{n}\left(\mathrm{H}_{2} \mathrm{O}\right)_{\mathrm{k}}$ clusters are quite close. The curves of $(1,1),(2,1)$ for MA and DMA show a similar tendency, in the case of $(2,2)$ at $k>2$, the affinities of the sulfuric acid to $(2,1)$ MA clusters are higher than those to $(2,1)$ DMA clusters. However, the values of the corresponding affinities to $(2,1)$ DMA- and MA-containing clusters averaged over the hydration number are quite close. This is a clear indication that at identical concentrations of MA and DMA, the impact of MA on formation stable sulfuric acid-water clusters will be quite close to that of the DMA.

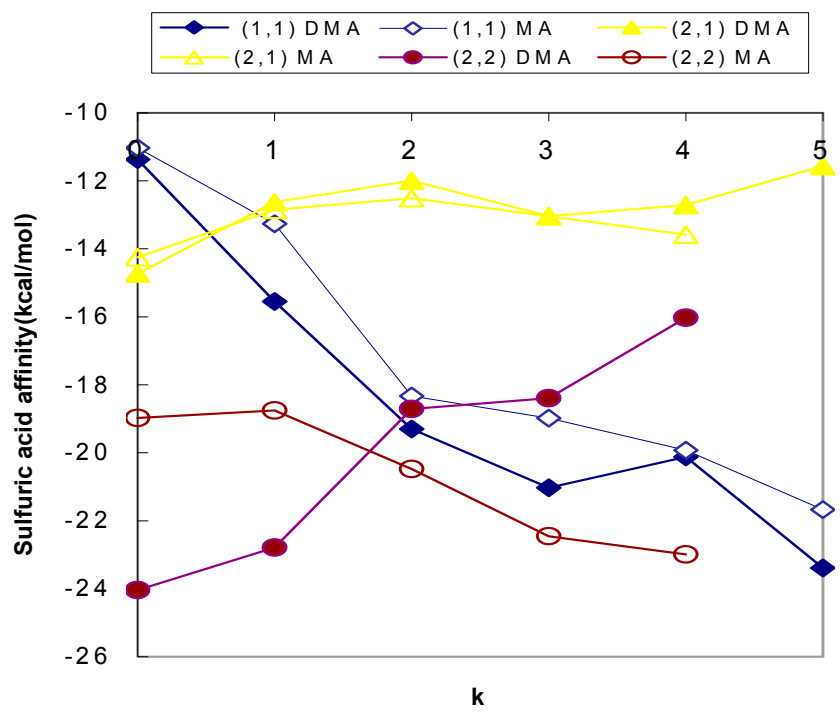

Figure 3. Standard conditions $(\mathrm{T}=298 \mathrm{~K}, \mathrm{P}=1013 \mathrm{KPa})$ Gibbs free energy changes associated with the formation of thermodynamically stable $\left(\mathrm{H}_{2} \mathrm{SO}_{4}\right)_{\mathrm{n}}\left(\mathrm{CH}_{3} \mathrm{NH}_{2}\right)_{\mathrm{m}}\left(\mathrm{H}_{2} \mathrm{O}\right)_{\mathrm{k}}$ and $\left(\mathrm{H}_{2} \mathrm{SO}_{4}\right)_{\mathrm{n}}\left(\left(\mathrm{CH}_{3}\right)_{2} \mathrm{NH}\right)_{\mathrm{m}}\left(\mathrm{H}_{2} \mathrm{O}\right)_{\mathrm{k}}$ clusters via the $\left(\mathrm{H}_{2} \mathrm{SO}_{4}\right)_{\mathrm{n}-1}\left(\mathrm{CH}_{3} \mathrm{NH}_{2}\right)_{\mathrm{m}}\left(\mathrm{H}_{2} \mathrm{O}\right)_{\mathrm{k}}+\left(\mathrm{H}_{2} \mathrm{SO}_{4}\right)<=>$ $\left(\mathrm{H}_{2} \mathrm{SO}_{4}\right)_{\mathrm{n}}\left(\mathrm{CH}_{3} \mathrm{NH}_{2}\right)_{\mathrm{m}}\left(\mathrm{H}_{2} \mathrm{O}\right)_{\mathrm{k}}$ and $\left(\mathrm{H}_{2} \mathrm{SO}_{4}\right)_{\mathrm{n}-1}\left(\left(\mathrm{CH}_{3}\right)_{2} \mathrm{NH}\right)_{\mathrm{m}}\left(\mathrm{H}_{2} \mathrm{O}\right)_{\mathrm{k}}+\left(\mathrm{H}_{2} \mathrm{SO}_{4}\right)<=>$ $\left(\mathrm{H}_{2} \mathrm{SO}_{4}\right)_{\mathrm{n}}\left(\left(\mathrm{CH}_{3}\right)_{2} \mathrm{NH}\right)_{\mathrm{m}}\left(\mathrm{H}_{2} \mathrm{O}\right)_{\mathrm{k}}$ reactions as functions of the hydration number k. DMA and MA refer to dimethyl- and methylamine, respectively. The data for ammonia and dimethylamine were adopted from $[26,61]$ and [22], respectively.

\subsubsection{Affinity of $\mathrm{CH}_{3} \mathrm{NH}_{2}$ to Nucleating $\left(\mathrm{H}_{2} \mathrm{SO}_{4}\right)_{\mathrm{m}}\left(\mathrm{CH}_{3} \mathrm{NH}_{2}\right)_{n}\left(\mathrm{H}_{2} \mathrm{O}\right)_{k}$ Clusters}

Table 3 presents enthalpies, entropies and Gibbs free energy changes describing the formation of $\left(\mathrm{H}_{2} \mathrm{SO}_{4}\right)_{n}\left(\mathrm{CH}_{3} \mathrm{NH}_{2}\right)_{\mathrm{m}}\left(\mathrm{H}_{2} \mathrm{O}\right)_{\mathrm{k}}$ clusters via the $\left(\mathrm{H}_{2} \mathrm{SO}_{4}\right)_{n}\left(\mathrm{CH}_{3} \mathrm{NH}_{2}\right)_{\mathrm{m}-1}\left(\mathrm{H}_{2} \mathrm{O}\right)_{\mathrm{k}}+\left(\mathrm{CH}_{3} \mathrm{NH}_{2}\right) \quad<=>$ $\left(\mathrm{H}_{2} \mathrm{SO}_{4}\right)_{n}\left(\mathrm{CH}_{3} \mathrm{NH}_{2}\right)_{\mathrm{m}}\left(\mathrm{H}_{2} \mathrm{O}\right)_{\mathrm{k}}$ reaction. As seen from Table 3, the MA affinities to nucleating $\left(\mathrm{H}_{2} \mathrm{SO}_{4}\right)_{\mathrm{n}}\left(\mathrm{CH}_{3} \mathrm{NH}_{2}\right)_{\mathrm{m}}\left(\mathrm{H}_{2} \mathrm{O}\right)_{\mathrm{k}}$ strongly depend on the molar fraction of $\mathrm{H}_{2} \mathrm{SO}_{4}$. In particular, they grow as the molar fraction of $\mathrm{H}_{2} \mathrm{SO}_{4}$ in nucleating cluster increases. The MA affinities to $\left(\mathrm{H}_{2} \mathrm{SO}_{4}\right)_{n}\left(\mathrm{CH}_{3} \mathrm{NH}_{2}\right)_{\mathrm{m}}\left(\mathrm{H}_{2} \mathrm{O}\right)_{\mathrm{k}}$ are much higher than $\mathrm{NH}_{3}$ affinities to $\left(\mathrm{H}_{2} \mathrm{SO}_{4}\right)_{n}\left(\mathrm{NH}_{3}\right)_{\mathrm{m}}\left(\mathrm{H}_{2} \mathrm{O}\right)_{\mathrm{k}}$ clusters and are close to the DMA affinities to $\left(\mathrm{H}_{2} \mathrm{SO}_{4}\right)_{n}\left(\left(\mathrm{CH}_{3}\right)_{2} \mathrm{NH}\right)_{\mathrm{m}}\left(\mathrm{H}_{2} \mathrm{O}\right)_{k}$.

The nature of the enhancement in the cluster stability due to the MA is same as that due to other common atmospheric bases such as $\mathrm{NH}_{3}$ and DMA. In particular, while the gas-phase MA acts, just like 
$\mathrm{NH}_{3}$ and DMA, as a highly reactive nucleation agent, the MA clustered with $\mathrm{H}_{2} \mathrm{SO}_{4}$ and $\mathrm{H}_{2} \mathrm{O}$ molecules enhances the affinity of the sulfuric acid to $\left(\mathrm{H}_{2} \mathrm{SO}_{4}\right)_{n}\left(\mathrm{CH}_{3} \mathrm{NH}_{2}\right)_{\mathrm{m}}\left(\mathrm{H}_{2} \mathrm{O}\right)_{\mathrm{k}}$ clusters being formed.

Table 3. Changes in enthalpies $\Delta \mathrm{H}\left(\mathrm{kcal} \mathrm{mol}^{-1}\right)$, entropies $\Delta \mathrm{S}\left(\mathrm{cal} \mathrm{mol}^{-1} \mathrm{~K}^{-1}\right)$, and Gibbs free energy $\Delta \mathrm{G}\left(\mathrm{kcal} \mathrm{mol}^{-1}\right)$ describing the affinity of $\mathrm{CH}_{3} \mathrm{NH}_{2}$ to nucleating $\left(\mathrm{H}_{2} \mathrm{SO}_{4}\right)_{\mathrm{n}}\left(\mathrm{CH}_{3} \mathrm{NH}_{2}\right)_{\mathrm{m}}\left(\mathrm{H}_{2} \mathrm{O}\right)_{\mathrm{k}}$ clusters at $298.15 \mathrm{~K}$ and pressure of $101.3 \mathrm{KPa}$.

\begin{tabular}{|c|c|c|c|}
\hline Reaction & $\Delta \mathbf{H}$ & $\Delta \mathbf{S}$ & $\Delta \mathbf{G}$ \\
\hline$\left(\mathrm{CH}_{3} \mathrm{NH}_{2}\right)+\left(\mathrm{CH}_{3} \mathrm{NH}_{2}\right) \Leftrightarrow=\left(\mathrm{CH}_{3} \mathrm{NH}_{2}\right)_{2}$ & -4.93 & -21.07 & 1.35 \\
\hline$\left(\mathrm{CH}_{3} \mathrm{NH}_{2}\right)_{2}+\left(\mathrm{CH}_{3} \mathrm{NH}_{2}\right)<=\left(\mathrm{CH}_{3} \mathrm{NH}_{2}\right)_{3}$ & -3.17 & -24.5 & 4.13 \\
\hline$\left(\mathrm{H}_{2} \mathrm{SO}_{4}\right)+\left(\mathrm{CH}_{3} \mathrm{NH}_{2}\right)<=\left(\mathrm{H}_{2} \mathrm{SO}_{4}\right)\left(\mathrm{CH}_{3} \mathrm{NH}_{2}\right)$ & -20.4 & -31.42 & -11.03 \\
\hline$\left(\mathrm{H}_{2} \mathrm{SO}_{4}\right)\left(\mathrm{H}_{2} \mathrm{O}\right)+\left(\mathrm{CH}_{3} \mathrm{NH}_{2}\right)<=\left(\mathrm{H}_{2} \mathrm{SO}_{4}\right)\left(\mathrm{CH}_{3} \mathrm{NH}_{2}\right)\left(\mathrm{H}_{2} \mathrm{O}\right)$ & -21.65 & -32.11 & -12.08 \\
\hline$\left(\mathrm{H}_{2} \mathrm{SO}_{4}\right)\left(\mathrm{H}_{2} \mathrm{O}\right)_{2}+\left(\mathrm{CH}_{3} \mathrm{NH}_{2}\right)<=\left(\mathrm{H}_{2} \mathrm{SO}_{4}\right)\left(\mathrm{CH}_{3} \mathrm{NH}_{2}\right)\left(\mathrm{H}_{2} \mathrm{O}\right)_{2}$ & -22.94 & -34.69 & -12.59 \\
\hline$\left(\mathrm{H}_{2} \mathrm{SO}_{4}\right)\left(\mathrm{H}_{2} \mathrm{O}\right)_{3}+\left(\mathrm{CH}_{3} \mathrm{NH}_{2}\right)<=\left(\mathrm{H}_{2} \mathrm{SO}_{4}\right)\left(\mathrm{CH}_{3} \mathrm{NH}_{2}\right)\left(\mathrm{H}_{2} \mathrm{O}\right)_{3}$ & -21.96 & -32.17 & -12.37 \\
\hline$\left(\mathrm{H}_{2} \mathrm{SO}_{4}\right)\left(\mathrm{H}_{2} \mathrm{O}\right)_{4}+\left(\mathrm{CH}_{3} \mathrm{NH}_{2}\right)<=\left(\mathrm{H}_{2} \mathrm{SO}_{4}\right)\left(\mathrm{CH}_{3} \mathrm{NH}_{2}\right)\left(\mathrm{H}_{2} \mathrm{O}\right)_{4}$ & -18.76 & -28.24 & -10.34 \\
\hline$\left(\mathrm{H}_{2} \mathrm{SO}_{4}\right)\left(\mathrm{H}_{2} \mathrm{O}\right)_{5}+\left(\mathrm{CH}_{3} \mathrm{NH}_{2}\right)<=\left(\mathrm{H}_{2} \mathrm{SO}_{4}\right)\left(\mathrm{CH}_{3} \mathrm{NH}_{2}\right)\left(\mathrm{H}_{2} \mathrm{O}\right)_{5}$ & -20.54 & -36.92 & -9.53 \\
\hline$\left(\mathrm{H}_{2} \mathrm{SO}_{4}\right)\left(\mathrm{CH}_{3} \mathrm{NH}_{2}\right)+\left(\mathrm{CH}_{3} \mathrm{NH}_{2}\right)<=\left(\mathrm{H}_{2} \mathrm{SO}_{4}\right)\left(\mathrm{CH}_{3} \mathrm{NH}_{2}\right)_{2}$ & -16.47 & -32.40 & -6.80 \\
\hline$\left(\mathrm{H}_{2} \mathrm{SO}_{4}\right)\left(\mathrm{CH}_{3} \mathrm{NH}_{2}\right)\left(\mathrm{H}_{2} \mathrm{O}\right)+\left(\mathrm{CH}_{3} \mathrm{NH}_{2}\right)<=\left(\mathrm{H}_{2} \mathrm{SO}_{4}\right)\left(\mathrm{CH}_{3} \mathrm{NH}_{2}\right)_{2}\left(\mathrm{H}_{2} \mathrm{O}\right)$ & -15.70 & -35.95 & -4.98 \\
\hline$\left(\mathrm{H}_{2} \mathrm{SO}_{4}\right)\left(\mathrm{CH}_{3} \mathrm{NH}_{2}\right)\left(\mathrm{H}_{2} \mathrm{O}\right)_{2}+\left(\mathrm{CH}_{3} \mathrm{NH}_{2}\right)<=\left(\mathrm{H}_{2} \mathrm{SO}_{4}\right)\left(\mathrm{CH}_{3} \mathrm{NH}_{2}\right)_{2}\left(\mathrm{H}_{2} \mathrm{O}\right)_{2}$ & -13.58 & -36.94 & -2.57 \\
\hline$\left(\mathrm{H}_{2} \mathrm{SO}_{4}\right)\left(\mathrm{CH}_{3} \mathrm{NH}_{2}\right)\left(\mathrm{H}_{2} \mathrm{O}\right)_{3}+\left(\mathrm{CH}_{3} \mathrm{NH}_{2}\right)<=\left(\mathrm{H}_{2} \mathrm{SO}_{4}\right)\left(\mathrm{CH}_{3} \mathrm{NH}_{2}\right)_{2}\left(\mathrm{H}_{2} \mathrm{O}\right)_{3}$ & -15.91 & -42.52 & -3.24 \\
\hline$\left(\mathrm{H}_{2} \mathrm{SO}_{4}\right)\left(\mathrm{CH}_{3} \mathrm{NH}_{2}\right)\left(\mathrm{H}_{2} \mathrm{O}\right)_{4}+\left(\mathrm{CH}_{3} \mathrm{NH}_{2}\right)<=\left(\mathrm{H}_{2} \mathrm{SO}_{4}\right)\left(\mathrm{CH}_{3} \mathrm{NH}_{2}\right)_{2}\left(\mathrm{H}_{2} \mathrm{O}\right)_{4}$ & -16.60 & -45.06 & -3.17 \\
\hline$\left(\mathrm{H}_{2} \mathrm{SO}_{4}\right)_{2}+\left(\mathrm{CH}_{3} \mathrm{NH}_{2}\right)<=>\left(\mathrm{H}_{2} \mathrm{SO}_{4}\right)_{2}\left(\mathrm{CH}_{3} \mathrm{NH}_{2}\right)$ & -31.65 & -40.13 & -19.69 \\
\hline$\left(\mathrm{H}_{2} \mathrm{SO}_{4}\right)_{2}\left(\mathrm{H}_{2} \mathrm{O}\right)+\left(\mathrm{CH}_{3} \mathrm{NH}_{2}\right)<=\left(\mathrm{H}_{2} \mathrm{SO}_{4}\right)_{2}\left(\mathrm{CH}_{3} \mathrm{NH}_{2}\right)\left(\mathrm{H}_{2} \mathrm{O}\right)$ & -28.47 & -34.41 & -18.21 \\
\hline$\left(\mathrm{H}_{2} \mathrm{SO}_{4}\right)_{2}\left(\mathrm{H}_{2} \mathrm{O}\right)_{2}+\left(\mathrm{CH}_{3} \mathrm{NH}_{2}\right) \Leftrightarrow=\left(\mathrm{H}_{2} \mathrm{SO}_{4}\right)_{2}\left(\mathrm{CH}_{3} \mathrm{NH}_{2}\right)\left(\mathrm{H}_{2} \mathrm{O}\right)_{2}$ & -29.11 & -35.91 & -18.41 \\
\hline$\left(\mathrm{H}_{2} \mathrm{SO}_{4}\right)_{2}\left(\mathrm{H}_{2} \mathrm{O}\right)_{3}+\left(\mathrm{CH}_{3} \mathrm{NH}_{2}\right)<=\left(\mathrm{H}_{2} \mathrm{SO}_{4}\right)_{2}\left(\mathrm{CH}_{3} \mathrm{NH}_{2}\right)\left(\mathrm{H}_{2} \mathrm{O}\right)_{3}$ & -26.53 & -29.64 & -17.70 \\
\hline$\left(\mathrm{H}_{2} \mathrm{SO}_{4}\right)_{2}\left(\mathrm{H}_{2} \mathrm{O}\right)_{4}+\left(\mathrm{CH}_{3} \mathrm{NH}_{2}\right)<=\left(\mathrm{H}_{2} \mathrm{SO} 4\right)_{2}\left(\mathrm{CH}_{3} \mathrm{NH}_{2}\right)\left(\mathrm{H}_{2} \mathrm{O}\right)_{4}$ & -24.05 & -27.87 & -15.74 \\
\hline$\left(\mathrm{H}_{2} \mathrm{SO}_{4}\right)_{2}\left(\mathrm{CH}_{3} \mathrm{NH}_{2}\right)+\left(\mathrm{CH}_{3} \mathrm{NH}_{2}\right)<=\left(\mathrm{H}_{2} \mathrm{SO}_{4}\right)_{2}\left(\mathrm{CH}_{3} \mathrm{NH}_{2}\right)_{2}$ & -20.13 & -28.86 & -11.52 \\
\hline$\left(\mathrm{H}_{2} \mathrm{SO}_{4}\right)_{2}\left(\mathrm{CH}_{3} \mathrm{NH}_{2}\right)\left(\mathrm{H}_{2} \mathrm{O}\right)+\left(\mathrm{CH}_{3} \mathrm{NH}_{2}\right)<=\left(\mathrm{H}_{2} \mathrm{SO}_{4}\right)_{2}\left(\mathrm{CH}_{3} \mathrm{NH}_{2}\right)_{2}\left(\mathrm{H}_{2} \mathrm{O}\right)$ & -20.94 & -33.69 & -10.89 \\
\hline$\left(\mathrm{H}_{2} \mathrm{SO}_{4}\right)_{2}\left(\mathrm{CH}_{3} \mathrm{NH}_{2}\right)\left(\mathrm{H}_{2} \mathrm{O}\right)_{2}+\left(\mathrm{CH}_{3} \mathrm{NH}_{2}\right)<=\left(\mathrm{H}_{2} \mathrm{SO}_{4}\right)_{2}\left(\mathrm{CH}_{3} \mathrm{NH}_{2}\right)_{2}\left(\mathrm{H}_{2} \mathrm{O}\right)_{2}$ & -19.07 & -28.61 & -10.54 \\
\hline$\left(\mathrm{H}_{2} \mathrm{SO}_{4}\right)_{2}\left(\mathrm{CH}_{3} \mathrm{NH}_{2}\right)\left(\mathrm{H}_{2} \mathrm{O}\right)_{3}+\left(\mathrm{CH}_{3} \mathrm{NH}_{2}\right)<=>\left(\mathrm{H}_{2} \mathrm{SO}_{4}\right)_{2}\left(\mathrm{CH}_{3} \mathrm{NH}_{2}\right)_{2}\left(\mathrm{H}_{2} \mathrm{O}\right)_{3}$ & -21.63 & -30.12 & -12.65 \\
\hline$\left(\mathrm{H}_{2} \mathrm{SO}_{4}\right)_{2}\left(\mathrm{CH}_{3} \mathrm{NH}_{2}\right)\left(\mathrm{H}_{2} \mathrm{O}\right)_{4}+\left(\mathrm{CH}_{3} \mathrm{NH}_{2}\right)<=>\left(\mathrm{H}_{2} \mathrm{SO}_{4}\right)_{2}\left(\mathrm{CH}_{3} \mathrm{NH}_{2}\right)_{2}\left(\mathrm{H}_{2} \mathrm{O}\right)_{4}$ & -20.4 & -26.21 & -12.58 \\
\hline
\end{tabular}

\subsection{Impacts of the MA on Formation of Nucleating Clusters under Atmospheric Conditions}

In this section, we use the new thermochemical data discussed in Section 3.1 to estimate the impact of MA on the formation of stable clusters containing $\mathrm{H}_{2} \mathrm{SO}_{4}$ and $\mathrm{H}_{2} \mathrm{O}$ under atmospheric conditions. Figure 4 presents the distributions of hydrated $\left(\mathrm{H}_{2} \mathrm{SO}_{4}\right)_{\mathrm{m}}\left(\mathrm{CH}_{3} \mathrm{NH}_{2}\right)_{n}$ clusters at an ambient temperature of $298.15 \mathrm{~K}$ and variable RH. As seen from Figure 4, most of $\left(\mathrm{H}_{2} \mathrm{SO}_{4}\right)_{1}\left(\mathrm{CH}_{3} \mathrm{NH}_{2}\right)_{1},\left(\mathrm{H}_{2} \mathrm{SO}_{4}\right)_{2}\left(\mathrm{CH}_{3} \mathrm{NH}_{2}\right)_{1}$ and $\left(\mathrm{H}_{2} \mathrm{SO}_{4}\right)_{2}\left(\mathrm{CH}_{3} \mathrm{NH}_{2}\right)_{2}$ clusters are hydrated under typical atmospheric conditions and, hence, the $\mathrm{RH}$ dependency of the cluster distributions is obvious. Only $<5 \%$ of $\left(\mathrm{H}_{2} \mathrm{SO}_{4}\right)_{1}\left(\mathrm{CH}_{3} \mathrm{NH}_{2}\right)_{1},<30 \%$ of $\left(\mathrm{H}_{2} \mathrm{SO}_{4}\right)_{2}\left(\mathrm{CH}_{3} \mathrm{NH}_{2}\right)$ and $<10 \%$ of $\left(\mathrm{H}_{2} \mathrm{SO}_{4}\right)_{2}\left(\mathrm{CH}_{3} \mathrm{NH}_{2}\right)_{2}$ remain unhydrated at the relatively low $\mathrm{RH}=$ $50 \%$. The fractions of the hydrated $\left(\mathrm{H}_{2} \mathrm{SO}_{4}\right)_{1}\left(\mathrm{CH}_{3} \mathrm{NH}_{2}\right)_{1},\left(\mathrm{H}_{2} \mathrm{SO}_{4}\right)_{2}\left(\mathrm{CH}_{3} \mathrm{NH}_{2}\right)_{1}$ and $\left(\mathrm{H}_{2} \mathrm{SO}_{4}\right)_{2}\left(\mathrm{CH}_{3} \mathrm{NH}_{2}\right)$ clusters grow quickly with as the $\mathrm{RH}$ increases, reaching over $90 \%$ at $\mathrm{RH}=70 \%$. At $\mathrm{RH}=90 \%$ almost all the $\left(\mathrm{H}_{2} \mathrm{SO}_{4}\right)_{1}\left(\mathrm{CH}_{3} \mathrm{NH}_{2}\right)_{1},\left(\mathrm{H}_{2} \mathrm{SO}_{4}\right)_{2}\left(\mathrm{CH}_{3} \mathrm{NH}_{2}\right)_{1}$ and $\left(\mathrm{H}_{2} \mathrm{SO}_{4}\right)_{2}\left(\mathrm{CH}_{3} \mathrm{NH}_{2}\right)$ clusters are hydrated.

The cluster distributions for $\left(\mathrm{H}_{2} \mathrm{SO}_{4}\right)_{1}\left(\mathrm{CH}_{3} \mathrm{NH}_{2}\right)_{1},\left(\mathrm{H}_{2} \mathrm{SO}_{4}\right)_{2}\left(\mathrm{CH}_{3} \mathrm{NH}_{2}\right)_{1}$ and $\left(\mathrm{H}_{2} \mathrm{SO}_{4}\right)_{2}\left(\mathrm{CH}_{3} \mathrm{NH}_{2}\right)_{2}$ have peaks at $k=2,3, k=2,3,4$, and $k=3,4$, respectively. The hydration of less populous 
$\left(\mathrm{H}_{2} \mathrm{SO}_{4}\right)_{1}\left(\mathrm{CH}_{3} \mathrm{NH}_{2}\right)_{2}$ is much weaker, however, fractions of hydrated $\left(\mathrm{H}_{2} \mathrm{SO}_{4}\right)_{1}\left(\mathrm{CH}_{3} \mathrm{NH}_{2}\right)_{2}$ clusters reach $\sim 15 \%, 25 \%$ and $40 \%$ at $\mathrm{R}=50 \%, 70 \%$ and $90 \%$, respectively. These considerations lead us to conclude that $\mathrm{RH}$ is clearly one of the key parameters controlling the $\left(\mathrm{H}_{2} \mathrm{SO}_{4}\right)_{\mathrm{m}}\left(\mathrm{CH}_{3} \mathrm{NH}_{2}\right)_{n}\left(\mathrm{H}_{2} \mathrm{O}\right)_{\mathrm{k}}$ formation and rates, at which the $\left(\mathrm{H}_{2} \mathrm{SO}_{4}\right){ }_{\mathrm{m}}\left(\mathrm{CH}_{3} \mathrm{NH}_{2}\right)_{\mathrm{n}}\left(\mathrm{H}_{2} \mathrm{O}\right)_{\mathrm{k}}$ clusters are formed under atmospheric conditions.

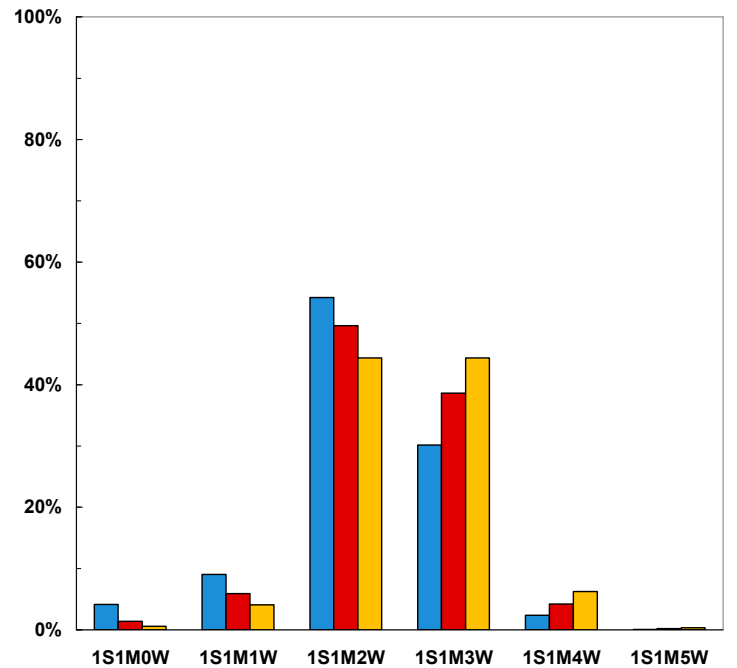

(a)

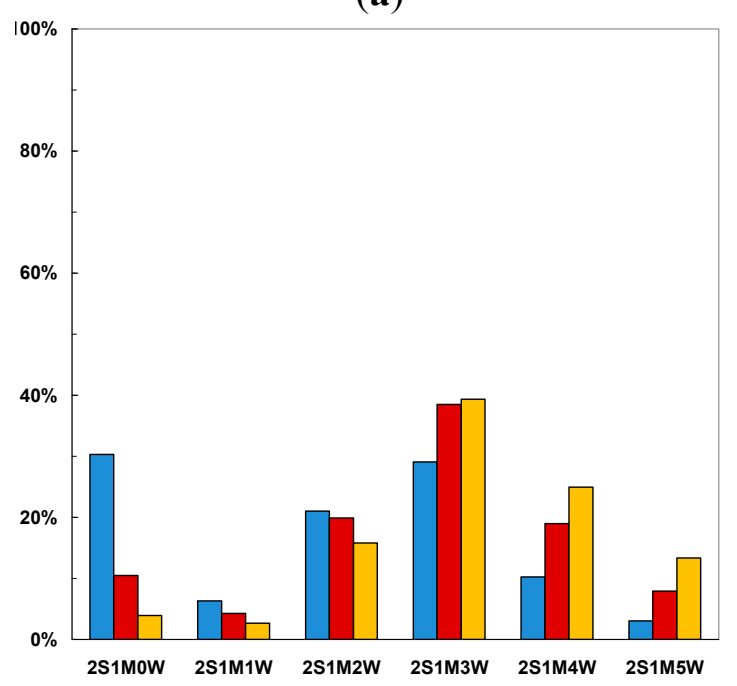

(c)

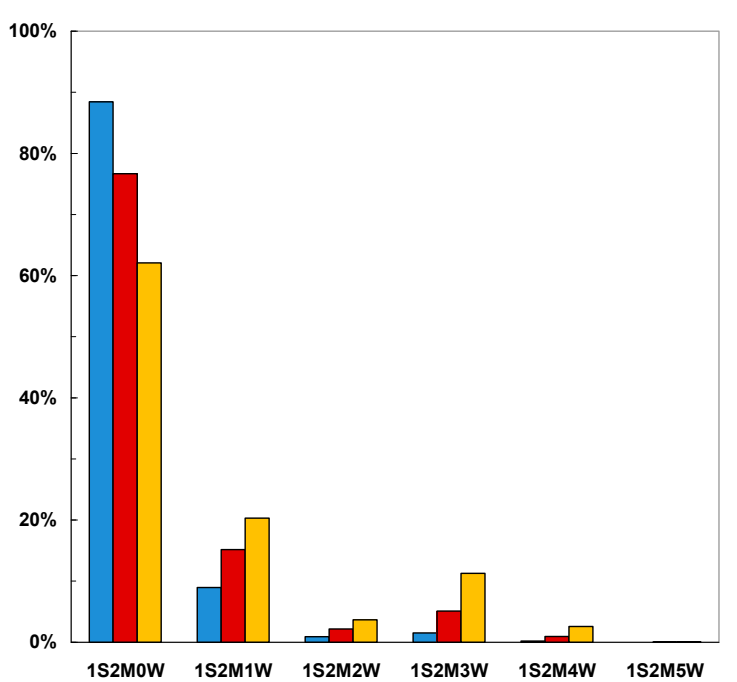

(b)

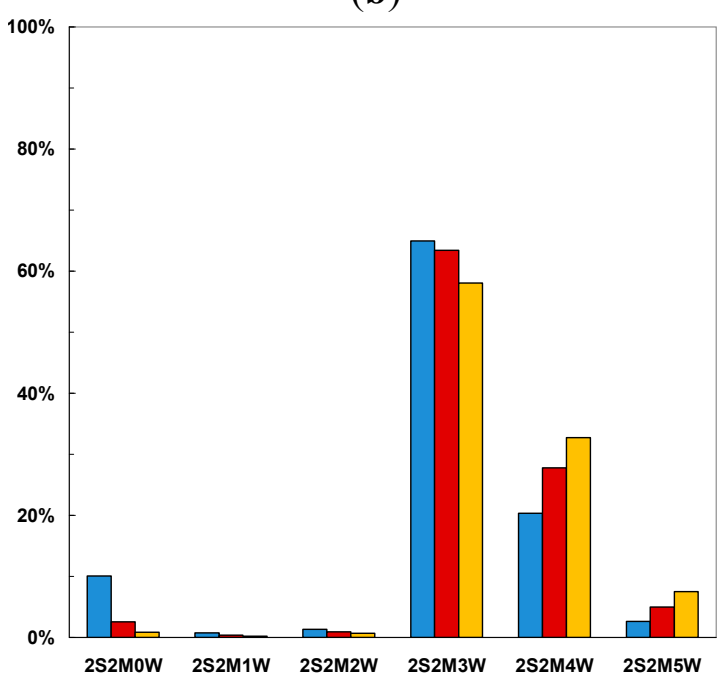

(d)

Figure 4. Fraction distribution of $\left(\mathrm{H}_{2} \mathrm{SO}_{4}\right)_{\mathrm{m}}\left(\mathrm{CH}_{3} \mathrm{NH}_{2}\right)_{\mathrm{n}}\left(\mathrm{H}_{2} \mathrm{O}\right)_{k}$ at ambient temperature $(298.15 \mathrm{~K})$ and variable RH. The abbreviation mSnMkW denotes $\left(\mathrm{H}_{2} \mathrm{SO}_{4}\right)_{\mathrm{m}}\left(\mathrm{CH}_{3} \mathrm{NH}_{2}\right)_{\mathrm{n}}\left(\mathrm{H}_{2} \mathrm{O}\right)_{\mathrm{k}}$ clusters. Blue, red and yellow bars represent $\mathrm{RH}$ of $50 \%, 70 \%$ and $90 \%$, respectively.

The formation of stable $\mathrm{H}_{2} \mathrm{SO}_{4}$ dimers is a critically important step in the base-enhanced new particle formation. The $\mathrm{H}_{2} \mathrm{SO}_{4}$ dimers consisting of two $\mathrm{H}_{2} \mathrm{SO}_{4}$, two base molecules and multiple water molecules are assumed to be both large and reactive enough to grow further via collisions with acids other than $\mathrm{H}_{2} \mathrm{SO}_{4}$ [62]. In this case, the production rates of $\left(\mathrm{H}_{2} \mathrm{SO}_{4}\right)_{2}(\text { amine })_{n}\left(\mathrm{H}_{2} \mathrm{O}\right)_{k}$ clusters are comparable to the new particle formation rates and can be used to estimate the maximum new particle production and its sensitivity to the concentration of base concentrations, $\mathrm{RH}$ and other relevant parameters. 
Figure 5 presents concentration ratios of ternary dimers (dimers containing MA or $\mathrm{NH}_{3}$ ) to binary $\mathrm{H}_{2} \mathrm{SO}_{4}-\mathrm{H}_{2} \mathrm{O}$ dimers characterizing the impacts of $\mathrm{MA}$ and $\mathrm{NH}_{3}$ on the formation of binary sulfuric acid-water clucters

(a) $\mathrm{T}=300 \mathrm{~K},\left[\mathrm{NH}_{3}\right]=1 \mathrm{ppb}$

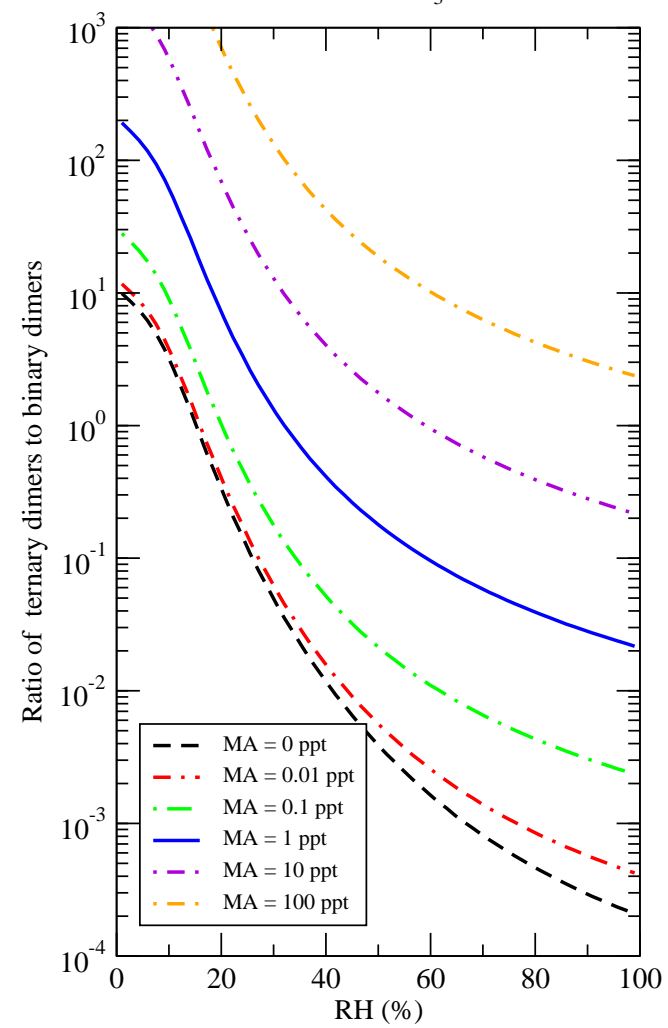

(b) $\mathrm{T}=270 \mathrm{~K},\left[\mathrm{NH}_{3}\right]=1 \mathrm{ppb}$

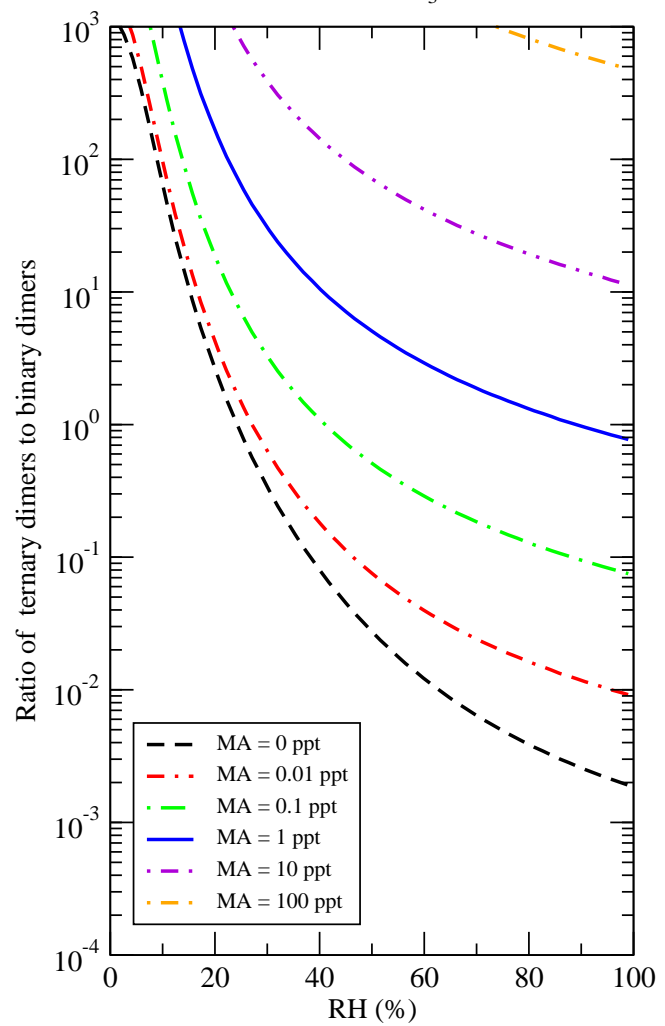

Figure 5. Concentration ratios of ternary dimers containing $\mathrm{MA}$ or $\mathrm{NH}_{3}$ to binary $\mathrm{H}_{2} \mathrm{SO}_{4}-\mathrm{H}_{2} \mathrm{O}$ dimers as a function of $\mathrm{RH}$. $\left[\mathrm{NH}_{3}\right]=1 \mathrm{ppb}$ is the background concentration of ammoniaMA- and $\mathrm{NH}_{3}$-enhanced cluster production are considered as simultaneous uncoupled processes. The data for ammonia were adopted from [26,61].

Two important observations can be made based on the comparison of the curves shown in Figure 5. First of all, the enhancement due to $1 \mathrm{ppb}$ of $\mathrm{NH}_{3}$ is either close to or smaller than that due to MA at ppt level. This is likely to indicate that accounting for the extended range of cluster sizes and compositions increases the difference in the stability of $\left(\mathrm{H}_{2} \mathrm{SO}_{4}\right)_{\mathrm{m}}\left(\mathrm{CH}_{3} \mathrm{NH}_{2}\right)_{n}\left(\mathrm{H}_{2} \mathrm{O}\right)_{\mathrm{k}}$ and $\left(\mathrm{H}_{2} \mathrm{SO}_{4}\right)_{\mathrm{m}}\left(\mathrm{NH}_{3}\right)_{\mathrm{n}}\left(\mathrm{H}_{2} \mathrm{O}\right)_{\mathrm{k}}$ clusters in the favor of $\left(\mathrm{H}_{2} \mathrm{SO}_{4}\right)_{\mathrm{m}}\left(\mathrm{CH}_{3} \mathrm{NH}_{2}\right)_{\mathrm{n}}\left(\mathrm{H}_{2} \mathrm{O}\right)_{\mathrm{k}}$ and that the difference in the cluster stability between $\left(\mathrm{H}_{2} \mathrm{SO}_{4}\right)_{\mathrm{m}}\left(\mathrm{CH}_{3} \mathrm{NH}_{2}\right)_{\mathrm{n}}\left(\mathrm{H}_{2} \mathrm{O}\right)_{\mathrm{k}}$ and $\left(\mathrm{H}_{2} \mathrm{SO}_{4}\right)_{\mathrm{m}}\left(\mathrm{NH}_{3}\right)_{\mathrm{n}}\left(\mathrm{H}_{2} \mathrm{O}\right)_{\mathrm{k}}$ is likely to be large enough to overcome the very large difference between atmospheric concentrations of [ $\mathrm{NH}_{3}$ ] ranging from $\sim 1$ to $10 \mathrm{ppb}$ and [MA] concentration ranging from a fraction of ppt to several ppt. The relative importance of ternary nucleation

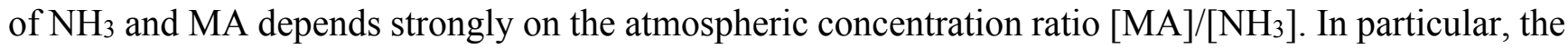
MA ternary nucleation begins to dominate over ternary nucleation of sulfuric acid, water and ammonia at $[\mathrm{MA}] /\left[\mathrm{NH}_{3}\right]>\sim 10^{-3}$. The conclusion about the relative importance of ternary $\mathrm{NH}_{3}$ and MA nucleation obtained based on extended thermodynamic data sets for MA with accounting for hydration slightly differs from that obtained in the earlier study [20]. Apparently, accounting for the wider range of cluster sizes and compositions leads to a moderate enhancement in the stability of $\mathrm{MA}-\mathrm{H}_{2} \mathrm{O}-\mathrm{H}_{2} \mathrm{SO}_{4}$ clusters compared to $\mathrm{NH}_{3}-\mathrm{H}_{2} \mathrm{O}-\mathrm{H}_{2} \mathrm{SO}_{4}$ ones. Another important detail is that $\left[\left(\mathrm{H}_{2} \mathrm{SO}_{4}\right)_{\mathrm{m}}\left(\mathrm{CH}_{3} \mathrm{NH}_{2}\right)_{n}\left(\mathrm{H}_{2} \mathrm{O}\right)_{\mathrm{k}}\right]$ dominate over 
$\left[\left(\mathrm{H}_{2} \mathrm{SO}_{4}\right)_{\mathrm{m}}\left(\mathrm{H}_{2} \mathrm{O}\right)_{\mathrm{k}}\right]$ under dry and low $\mathrm{RH}$ conditions only. For example, while at $\mathrm{RH}=20 \%$ [MA] at sub-ppt level is enough to reach $\left[\left(\mathrm{H}_{2} \mathrm{SO}_{4}\right)_{\mathrm{m}}\left(\mathrm{CH}_{3} \mathrm{NH}_{2}\right)_{\mathrm{n}}\left(\mathrm{H}_{2} \mathrm{O}\right)_{\mathrm{k}}\right] /\left[\left(\mathrm{H}_{2} \mathrm{SO}_{4}\right)_{\mathrm{m}}\left(\mathrm{H}_{2} \mathrm{O}\right)_{\mathrm{k}}\right]$ ratio of 10 , much higher [MA] exceeding $10 \mathrm{ppt}$ is needed in order to reach the same $\left[\left(\mathrm{H}_{2} \mathrm{SO}_{4}\right)_{\mathrm{m}}\left(\mathrm{CH}_{3} \mathrm{NH}_{2}\right)_{\mathrm{n}}\left(\mathrm{H}_{2} \mathrm{O}\right)_{\mathrm{k}}\right] /\left[\left(\mathrm{H}_{2} \mathrm{SO}_{4}\right)_{\mathrm{m}}\left(\mathrm{H}_{2} \mathrm{O}\right)_{\mathrm{k}}\right]$ at $\mathrm{RH}=60 \%$. The impact of the ambient temperature on the enhancement due to the MA is also very strong. In particular, the enhancement due to the MA increases with the decreasing ambient temperature. For example, the $\left[\left(\mathrm{H}_{2} \mathrm{SO}_{4}\right)_{\mathrm{m}}\left(\mathrm{CH}_{3} \mathrm{NH}_{2}\right)_{\mathrm{n}}\left(\mathrm{H}_{2} \mathrm{O}\right)_{\mathrm{k}}\right] /$ $\left[\left(\mathrm{H}_{2} \mathrm{SO}_{4}\right)_{\mathrm{m}}\left(\mathrm{H}_{2} \mathrm{O}\right)_{\mathrm{K}}\right]$ ratios at $\mathrm{T}=300 \mathrm{~K}$ and $\mathrm{T}=270 \mathrm{~K}$ differ by more than two orders of magnitude in the favor of the $\left[\left(\mathrm{H}_{2} \mathrm{SO}_{4}\right)_{\mathrm{m}}\left(\mathrm{CH}_{3} \mathrm{NH}_{2}\right)_{n}\left(\mathrm{H}_{2} \mathrm{O}\right)_{\mathrm{k}}\right] /\left[\left(\mathrm{H}_{2} \mathrm{SO}_{4}\right)_{\mathrm{m}}\left(\mathrm{H}_{2} \mathrm{O}\right)_{\mathrm{k}}\right]$ at $\mathrm{T}=270 \mathrm{~K}$. As it may be seen from Figure 5, the equilibrium concentrations of $\left(\mathrm{H}_{2} \mathrm{SO}_{4}\right)$ clusters containing MA and strength of the enhancing effect of the MA depends on the RH and the ambient temperature. Low RH and low ambient temperatures are clearly the favorable conditions at which the strongest effect of MA on the formation of stable binary $\mathrm{H}_{2} \mathrm{SO}_{4}-\mathrm{H}_{2} \mathrm{O}$ clusters is achieved.

\section{Conclusions}

In this paper, the enhancement in production of stable sulfuric acid clusters due to methylamine has been studied and the lower limit of the impact of methylamines on nucleation in the Earth's atmosphere has been investigated. The presents study leads us to the following important conclusions:

(a) $\left(\mathrm{H}_{2} \mathrm{SO}_{4}\right)_{\mathrm{m}}\left(\mathrm{CH}_{3} \mathrm{NH}_{2}\right)_{\mathrm{n}}\left(\mathrm{H}_{2} \mathrm{O}\right)_{\mathrm{k}}$ clusters are strongly hydrated under typical atmospheric conditions and, thus, $\left(\mathrm{H}_{2} \mathrm{SO}_{4}\right)_{\mathrm{m}}\left(\mathrm{CH}_{3} \mathrm{NH}_{2}\right)_{\mathrm{n}}\left(\mathrm{H}_{2} \mathrm{O}\right)_{\mathrm{k}}$ formation rates are $\mathrm{RH}$-dependent. The effect of the MA depends strongly on the $\mathrm{RH}$ and the ambient temperature. Low $\mathrm{RH}$ and low ambient temperatures are clearly the favorable conditions, at which the strongest effect of MA on the formation of stable binary $\mathrm{H}_{2} \mathrm{SO}_{4}-\mathrm{H}_{2} \mathrm{O}$ clusters is achieved. While the enhancing effect is very strong at low $\mathrm{RH}$, it decreases quickly as $\mathrm{RH}$ is growing;

(b) At the identical concentrations of MA and DMA, the enhancement in the production of stable $\left(\mathrm{H}_{2} \mathrm{SO}_{4}\right)_{\mathrm{m}}\left(\mathrm{H}_{2} \mathrm{O}\right)_{\mathrm{k}}$ due to MA is close to that due to DMA;

(c) The MA ternary nucleation begins to dominate over ternary nucleation of sulfuric acid, water and ammonia at $[\mathrm{MA}] /\left[\mathrm{NH}_{3}\right]>\sim 10^{-3}$.

The new data on thermochemistry of ternary nucleation of MA obtained in the present study can be used to solve a number of problems related to the physics and chemistry of gas-to-cluster conversion, including calculations of temperature- and concentration-dependent free energies and reaction constants for a number of reactions, equilibrium and steady-state concentrations of nucleating clusters, gas-clusternanoparticle phase transitions in multicomponent systems, and can be utilized directly for the development of quantum-constrained nucleation models, in which the first nucleation steps are treated using the quantum approach. Further research will be focused on the incorporation of obtained data into the framework of a kinetic nucleation model and comparison of the simulation results with atmospheric observations.

\section{Acknowledgements}

The support of this study by the Russian Ministry of Education under the State Research Task Program (Grant No. 14-04/0110), the National Science Foundation of the USA, the National Natural 
Science Foundation of China (Grant 41375133) and the Science Foundation of Chinese Research Academy of Environmental Sciences (Grant 2012-YSKY-15) is gratefully acknowledged.

\section{Author Contributions}

A.B. Nadykto, Y. Xu, F. Yu, E. Nazarenko and J. Herb conceived the idea. J. Herb, E. Nazarenko, A.B. Nadykto and Y. Xu carried out computations. A.B. Nadykto, J. Herb, E. Nazarenko, F. Yu and Y. Xu performed the data analysis. A.B. Nadykto wrote the paper. All authors have read and approved the final manuscript.

\section{References}

1. Zhang, R. Getting to the Critical Nucleus of Aerosol Formation. Science 2010, 328, 1366-1367.

2. Yu, F.; Luo, G. Simulation of Particle Size Distribution with a Global Aerosol Model: Contribution of Nucleation to Aerosol and CCN Number Concentrations. Atmos. Chem. Phys. 2009, 9, 7691-7710.

3. Paasonen, P.; Asmi, A.; Petäjä, T.; Kajos, M.K.; Äijälä, M.; Junninen, H.; Holst, T.; Abbatt, J.P.D.; Arneth, A. ; Birmili, W. et al. Warming-induced increase in aerosol number concentration likely to moderate climate change. Nature Geosci. 2013, 6, 438-442.

4. Yu, F.; Hallar, G. Difference in Particle Formation at a Mountain-Top Location during the Spring and Summer: Implications for the Role of Sulfuric Acid and Organics in Nucleation. J. Geophys. Res. 2014, 119, 12-246.

5. Knaapen, M.; Borm, P.; Albrecht, C.; Schins, R.P.F. Inhaled Particles and Lung Cancer. Part A: Mechanisms. Int. J. Cancer 2004, 109, 799-809.

6. Saxon, D.; Diaz-Sanchez, D. Air pollution and allergy: You are what you breathe. Nature Immunol. 2005, 6, 223-226.

7. Zhang, R.; Khalizov, A.F.; Wang, L.; Hu, M.; Wen, X. Nucleation and Growth of Nanoparticles in the Atmosphere. Chem. Rev. 2012, 112, doi:10.1021/cr2001756.

8. Flood, H. Tröpfchenbildung in übersättigten Äthylalkohol-wasserdampfgemischen. Z Phys Chem A 1934, 170, 286-295.

9. Reiss, H. The Kinetics of Phase Transitions in Binary Systems. J. Chem. Phys. 1950, 18, 840-849.

10. Kulmala, M.; Pirjola, L.; Makela J.M. Stable Sulphate Clusters as a Source of New Atmospheric Particles. Nature 2000, 404, 66-69.

11. Yu, F.; Turco, R.P. Ultrafine Aerosol Formation via Ion-Mediated Nucleation. Geophys. Res. Lett. 2000, 27, 883-886.

12. Yu, F.; Luo, G. Effect of Solar Variations on Particle Formation and Cloud Condensation Nuclei. Environ. Res. Lett. 2014, 9, 045004.

13. Kirkby, J.; Curtius, J.; Almeida, J.; Dunne, E.; Duplissy, J.; Ehrhart, S.; Franchin, A.; Gagné, S.; Ickes, L.; Kürten, A. et al. Role of Sulphuric Acid, Ammonia and Galactic Cosmic Rays in Atmospheric Aerosol Nucleation. Nature 2011, 476, 429-433.

14. Napari, I.; Noppel, M.; Vehkamaki, H.; Kulmala, M. An Improved Model for Ternary Nucleation of Sulfuric Acid-ammonia-water. J. Chem. Phys. 2002, 116, doi: 10.1063/1.1450557. 
15. Anttila, T.; Vehkamaki, H.; Napari, I.; Kulmala, M. Effect of Ammonium Bisulphate Formation on Atmospheric Water-sulphuric Acid-ammonia Nucleation. Boreal Environ. Res. 2005, 10, 511-523.

16. Yu, F. Effect of Ammonia on New Particle Formation: A Kinetic $\mathrm{H}_{2} \mathrm{SO}_{4}-\mathrm{H}_{2} \mathrm{O}-\mathrm{NH}_{3}$ Nucleation Model Constrained by Laboratory Measurements. J. Geophys. Res. 2006, 111, doi: 10.1029/ $2005 \mathrm{jd} 005968$.

17. Ball, S.M.; Hanson, D.R.; Eisele, F.L.; McMurry, P.H. Laboratory Studies of Particle Nucleation: Initial Results for $\mathrm{H}_{2} \mathrm{SO}_{4}, \mathrm{H}_{2} \mathrm{O}$, and $\mathrm{NH}_{3}$ Vapors. J. Geophys. Res. 1999, 104, 23709-23718.

18. Benson, D.R.; Erupe, M.E.; Lee, S.-H. Laboratory-measured $\mathrm{H}_{2} \mathrm{SO}_{4}-\mathrm{H}_{2} \mathrm{O}-\mathrm{NH}_{3}$ Ternary Homogeneous Nucleation Rates: Initial Observations. Geophys. Res. Lett. 2009, 36, L15818.

19. Loukonen, V.; Kurten, T.; Ortega, I.K; Vehkamaki, H.; Padua, A.A.H.; Sellegri, K.; Kulmala, M. Enhancing Effect of Dimethylamine in Sulfuric Acidnucleation in the Presence of Water-a Computational Study. Atmos. Chem. Phys. 2010, 10, 4961-4974.

20. Nadykto, A.B.; Yu, F.; Jakovleva, M.; Herb, J.; Xu, Y. Amines in the Earth's Atmosphere: A Density Functional Theory Study of the Thermochemistry of Pre-Nucleation Clusters. Entropy 2011, 13, 554-569.

21. Ortega, I.K.; Kupiainen, O.; Kurten, T.; Olenius, T.; Wilkman, O.; McGrath, M.J.; Loukonen, V.; Vehkamaki, H. From quantum chemical formation free energies to evaporation rates. Atmos. Chem. Phys. 2012, 12, 225-235, doi:10.5194/acp-12-225-2012.

22. Nadykto, A.B.; Herb, J.; Yu, F.; Xu, Y. Enhancement in the Production of Nucleating Clusters due to Dimethylamine and Large Uncertainties in the Thermochemistry of Amine-enhanced Nucleation. Chem. Phys. Lett. 2014, 609, 42-49.

23. Almeida, J.; Schobesberger, S.; Kürten, A.; Ortega, I.K.; Kupiainen-Määttä, O.; Praplan, A.P.; Adamov, A.; Amorim, A.; Bianchi, F.; Breitenlechner, M. et al. Molecular Understanding of Sulphuric Acid-amine Particle Nucleation in the Atmosphere. Nature 2013, 502, 359-363.

24. Zhang, R.Y.; Suh, I.; Zhao, J.; Zhang, D.; Fortner, E.C.; Tie, X.X.; Molina, L.T.; Molina, M.J., Atmospheric New Particle Formation Enhanced by Organic acids. Science 2004, 304, 1487-1490.

25. Nadykto, A.B.; Yu, F. Strong Hydrogen Bonding between Atmospheric Nucleation Precursors and Common Organics. Chem. Phys. Lett. 2007, 435, doi:10.1016/j.cplett.2006.12.050.

26. Nadykto, A.B.; Al Natsheh, A.; Yu, F.; Mikkelsen, K.V.; Herb, J. Computational Quantum Chemistry: A New Approach to Atmospheric Nucleation. Adv. Quant. Chem. 2008, 55, 449-478.

27. Xu, Y.-S.; Nadykto, A.B.; Yu, F.; Jiang, L.; Wang, L. Formation and Properties of Hydrogenbonded Complexes of Common Organic Oxalic Acid with Atmospheric Nucleation Precursors. J. Mol. Struct. Theochem. 2010, 951, 28-33.

28. Xu, Y.-S.; Nadykto, A.B.; Yu, F.; Jiang, L.; Wang, L. Interaction between Common Organic Acids and Trace Nucleation Species in the Earth's Atmosphere. J. Phys. Chem. A 2010, 114, doi: $10.1021 /$ jp9068575.

29. Zhao, J.; Khalizov, A.; Zhang, R.; McGraw, R. Hydrogen-bonding Interaction in Molecular Complexes and Clusters of Aerosol Nucleation Precursors. J. Phys. Chem. A 2009, 113, 680-689.

30. Xu, W.; Zhang, R. A Theoretical Study of Hydrated Molecular Clusters of Amines and Dicarboxylic Acids. J. Chem. Phys. 2013, 139, 064312. 
31. Elm, J.; Kurtén, T.; Bilde, M.; Mikkelsen, K.V. Molecular Interaction of Pinic Acid with Sulfuric Acid: Exploring the Thermodynamic Landscape of Cluster Growth. J. Phys. Chem. A 2014, 118, 7892-7900.

32. Elm, J.; Jørgensen, S.; Bilde, M.; Mikkelsen, K.V. Ambient Reaction Kinetics of Atmospheric Oxygenated Organics with the $\mathrm{OH}$ Radical: A Computational Methodology Study. Phys. Chem. Chem. Phys. 2013, 15, 9636-9645.

33. Ge, X.; Wexler, A.S.; Clegg, S.L. Atmospheric Amines-Part II. Thermodynamic Properties and Gas/Particle Partitioning. Atmos. Environ. 2010, 45, 561-577.

34. You, Y.; Kanawade, V.P.; de Gouw, J.A.; Guenther, A.B.; Madronich, S.; Sierra-Hernández, M.R.; Lawler, M.; Smith, J.N.; Takahama , S.; Ruggeri ,G. et al. Atmospheric amines and ammonia measured with a chemical ionization mass spectrometer (CIMS). Atmos. Chem. Phys. 2014, 14, 12181-12194.

35. Hertel, O.; Reis, S.; Skjøth, C.A.; Bleeker, A.; Harrison, R.; Cape, J.N.; Fowler, D.; Skiba, U.; Simpson ,D.; Jickells ,T.; Baker, A.; Kulmala, M. et al. Nitrogen Processes in the Atmosphere. Philos. Trans. R. Soc. 2013, 368, 177-207.

36. Lee, D.; Wexler, A.S. Atmospheric amines-Part III: Photochemistry and Toxicity. Atmos. Environ. 2013, 71, 95-103.

37. Ge, X.; Wexler, A.S.; Clegg, S.L. Atmospheric Amines-Part I. a Review. Atmos. Environ. 2010, $45,524-546$.

38. Finlayson-Pitts, B.J.; Pitts, J.N. Chemistry of the Upper and Lower Atmosphere: Theory, Experiments, and Applications; Academic Press: San Diego, CA, USA, 2000.

39. Nielsen, C.J.; D’Anna, B.; Karl, M.; Aursnes, M.; Boreave, A.; Bossi, R.; Bunkan, A.J.C.; Glasius, M.; Hallquist, M.; Hansen, A.M.K. et al. Summary Report: Photo-Oxidation of Methylamine; Dimethylamine and Trimethylamine CLIMIT Project no. 201604; Norwegian Institute for Air Research: Kjeller, Norway, 2011.

40. Akyüz, M. Simultaneous Determination of Aliphatic and Aromatic Amines in Indoor and Outdoor Air Samples by Gas Chromatography-Mass Spectrometry. Talanta 2007, 71, 486-492.

41. Dawson, M.L.; Perraud, V.; Gomez, A.; Arquero, K.D.; Ezell, M.J.; Finlayson-Pitts, B.J. Measurement of Gas-phase Ammonia and Amines in Air by Collection onto an Ion Exchange Resin and Analysis by Ion Chromatography. Atmos. Meas. Tech. Discuss. 2014, 7, 1573-1602.

42. Yu, H.; Lee, S.-H. A Chemical Ionization Mass Spectrometer for the Detection of Atmospheric Amines. Environ. Chem. 2012, 9, 190-201.

43. Benson, D.R.; Markovich, A.; Al-Refai, M.; Lee, S.-H. A Chemical Ionization Mass Spectrometer for Ambient Measurements of Ammonia. Atmos. Meas. Tech. 2010, 3, 1075-1087.

44. Erupe, M.E.; Benson, D.R.; Li, J.; Young, L.-H.; Verheggen, B.; Al-Refai, M.; Tahboub, O.; Cunningham, V.; Frimpong, F.; Viggiano, A.A.; Lee, S.-H. Correlation of Aerosol Nucleation Rate with Sulfuric Acid and Ammonia in Kent Ohio: an Atmospheric Observation. J. Geophys. Res. 2010, 115, D23216.

45. Nowak, J.B.; Neuman, J.A.; Bahreini, R.; Brock, C.A.; Middlebrook, A.M.; Wollny, A.G.; Holloway, J.S.; Peischl, J.; Ryerson, T.B.; Fehsenfeld, F.C. Airborne Observations of Ammonia and Ammonium Nitrate Formation over Houston, Texas. J. Geophys. Res. 2010, 115, doi: 10.1029/2010JD014195. 
46. Yu, H.; McGraw, R.; Lee, S.-H. Effects of Amines on Formation of Sub-3nm Particles and their Subsequent Growth. Geophys. Res. Lett. 2012, 39, L02807.

47. Erupe, M.E.; Viggiano, A.A.; Lee, S.-H. The Effect of Trimethylamine on Atmospheric Nucleation Involving $\mathrm{H}_{2} \mathrm{SO}_{4}$. Atmos. Chem. Phys. 2011, 11, 4767-4775.

48. Berndt, T.; Sipilä, M.; Stratmann, F.; Petäjä, T.; Vanhanen, J.; Mikkilä, J.; Patokoski, J.; Taipale, R.; Mauldin, R.L.; Kulmala, M. Enhancement of Atmospheric $\mathrm{H}_{2} \mathrm{SO}_{4} / \mathrm{H}_{2} \mathrm{O}$ Nucleation: Organic Oxidation Products versus Amines. Atmos. Chem. Phys. 2014, 14, 751-764.

49. Zollner, J.H.; Glasoe, W.A.; Panta, B.; Carlson, K.K.; McMurry, P.H.; Hanson, D.R. Sulfuric Acid Nucleation: Power Dependencies, Variation with Relative Humidity, and Effect of Bases. Atmos. Chem. Phys. 2012, 12, 4399-4411.

50. Jen, C.N.; McMurry, P.H.; Hanson, D.R. Stabilization of Sulfuric Acid Dimers by Ammonia, Methylamine, Dimethylamine, and Trimethylamine. J. Geophys. Res. Atmos. 2014, 119, 7502-7514.

51. Qiu, C.; Zhang, R. Multiphase Chemistry of Atmospheric Amines. Phys. Chem. Chem. Phys. 2013, $15,5738-5752$.

52. Al Natsheh, A.; Nadykto, A.B.; Mikkelsen, K.V.; Yu, F.; Ruuskanen, J. Quantum Nature of the Sign Preference in Ion-Induced Nucleation. J. Phys. Rev. Lett. 2006, 96, 125701.

53. Nadykto, A.B.; Yu, F.; Herb, J. Towards Understanding the Sign Preference in Binary Atmospheric Nucleation. Phys. Chem. Chem. Phys. 2008, 10, 7073-7078.

54. Du, H.; Nadykto, A.B.; Yu, F. Quantum-mechanical Solution to Fundamental Problems of Classical Theory of Water Vapor Nucleation. Phys. Rev. E 2009, 79, 021604.

55. Hanson, D.R.; Lovejoy, E.R. Measurement of the Thermodynamics of the Hydrated Dimer and Trimer of Sulfuric Acid. J. Phys. Chem. A 2006, 110, 9525-9528.

56. Froyd, K.D.; Lovejoy, E.R. Experimental Thermodynamics of Cluster Ions Composed of $\mathrm{H}_{2} \mathrm{SO}_{4}$ and $\mathrm{H}_{2} \mathrm{O}$ Positive Ions. J. Phys. Chem. A 2003, 107, 9812-9824.

57. Temelso, B.; Ngoc Phan, T.; Shields, G.C. Computational Study of the Hydration of Sulfuric Acid Dimers: Implications for Acid Dissociation and Aerosol Formation. J. Phys. Chem. A 2012, 116, 9745-9758.

58. Bustos, D.J.; Temelso, B.; Shields, G.C. Hydration of the Sulfuric Acid-Methylamine Complex and Implications for Aerosol Formation. J. Phys. Chem. A 2014, 118, 7430-7441.

59. Husar, D.E.; Temelso, B.; Ashworth, A.L.; Shields, G.C. Hydration of the Bisulfate ion: Atmospheric Implications. J. Phys. Chem. A 2012, 116, 5151-5163.

60. Gaussian 09; Revision D.01; Frisch, M.J.; Trucks, G.W.; Schlegel, H.B.; Scuseria, G.E.; Robb, M.A.; Cheeseman, J.R.; Scalmani, G.; Barone, V.; Mennucci, B.; Petersson, G.A.; Nakatsuji, H.; Caricato, M.; Li, X.; Hratchian, H.P.; Izmaylov, A.F.; Bloino, J.; Zheng, G.; Sonnenberg, J.L.; Hada, M.; Ehara, M.; Toyota, K.; Fukuda, R.; Hasegawa, J.; Ishida, M.; Nakajima, T.; Honda, Y.; Kitao, O.; Nakai, H.; Vreven, T.; Montgomery, J.A., Jr.; Peralta, J.E.; Ogliaro, F.; Bearpark, M.; Heyd, J.J.; Brothers, E.; Kudin, K.N.; Staroverov, V.N.; Kobayashi, R.; Normand, J.; Raghavachari, K.; Rendell, A.; Burant, J.C.; Iyengar, S.S.; Tomasi, J.; Cossi, M.; Rega, N.; Millam, J.M.; Klene, M.; Knox, J.E.; Cross, J.B.; Bakken, V.; Adamo, C.; Jaramillo, J.; Gomperts, R.; Stratmann, R.E.; Yazyev, O.; Austin, A.J.; Cammi, R.; Pomelli, C.; Ochterski, J.W.; Martin, R.L.; Morokuma, K.; Zakrzewski, V.G.; Voth, G.A.; Salvador, P.; Dannenberg, J.J.; Dapprich, S.; Daniels, A.D.; Farkas, Ö.; Foresman, J.B.; Ortiz, J.V.; Cioslowski, J.; Fox, D.J. Gaussian, Inc.: Wallingford, CT, USA, 2009. 
61. Herb, J.; Nadykto, A.B.; Yu, F. Large Ternary Hydrogen-bonded Pre-nucleation Clusters in the Earth's Atmosphere. Chem. Phys. Lett. 2011, 518, 7-14.

62. Paasonen, P.; Olenius, T.; Kupiainen, O.; Kurten, T.; Petaja, T.; Birmili, W.; Hamed, A.; Hu, M.; Huey, L.G.; Plass-Duelmer, C.; et al. On the Formation of Sulphuric Acid-Amine Clusters in Varying Atmospheric Conditions and its Influence on Atmospheric new Particle Formation. Atmos. Chem. Phys. 2012, 12, 9113-9133.

(C) 2015 by the authors; licensee MDPI, Basel, Switzerland. This article is an open access article distributed under the terms and conditions of the Creative Commons Attribution license (http://creativecommons.org/licenses/by/4.0/). 